\title{
The stability of a flexible cantilever in viscous channel flow
}

\author{
Julien Cisonni ${ }^{\mathrm{a}, *}$, Anthony D. Lucey ${ }^{\mathrm{a}}$, Novak S. J. Elliott ${ }^{\mathrm{a}, \mathrm{b}}$, Matthias Heil ${ }^{\mathrm{c}}$ \\ ${ }^{a}$ Fluid Dynamics Research Group, Department of Mechanical Engineering, Curtin University, Perth, Australia \\ ${ }^{b}$ Grupo de Investigación de la Dinámica de Fluidos, Xe' Lajuj No’j, Quetzaltenango, Guatemala \\ ${ }^{c}$ School of Mathematics, University of Manchester, Manchester, United Kingdom
}

\begin{abstract}
Most studies of the flow-induced flutter instability of a flexible cantilever have assumed inviscid flow because of the high flow speeds and the large scale of the structures encountered in the wide range of applications of this fluidstructure interaction (FSI) system. However, for instance, in the fields of energy harvesting and biomechanics, low flow speeds and small- and micro-scale systems can give relatively low Reynolds numbers so that fluid viscosity needs to be explicitly accounted for to provide reliable predictions of channel-immersed-cantilever stability. In this study, we employ a numerical model coupling the Navier-Stokes equations and a one-dimensional elastic beam model. We conduct a parametric investigation to determine the conditions leading to flutter instability of a slender flexible cantilever immersed in two-dimensional viscous channel flow for Reynolds numbers lower than 1000. The large set of numerical simulations carried out allows predictions of the influence of decreasing Reynolds numbers and of the cantilever confinement on the single-mode neutral stability of the FSI system and on the pre- and post-critical cantilever motion. This model's predictions are also compared to those of a FSI model containing a two-dimensional solid model in order to assess, primarily, the effect of the cantilever slenderness in the simulations. Results show that an increasing contribution of viscosity to the hydrodynamic forces significantly alters the instability boundaries. In general, a decrease in Reynolds number is predicted to produce a stabilisation of the FSI system, which is more pronounced for high fluid-to-solid mass ratios. For particular fluid-to-solid mass ratios, viscous effects can lower the critical velocity and lead to a change in the first unstable structural mode. However, at constant Reynolds number, the effects of viscosity on the system stability are diminished by the confinement of the cantilever, which strengthens the importance of flow inertia.
\end{abstract}

Keywords: Fluid-structure interaction, Flexible cantilever, Flutter instability, Viscous channel flow

\section{Introduction}

The flow-induced flutter instability of a flexible cantilever is a fundamental fluid-structure interaction (FSI) problem with applications in many long-established fields of engineering, ranging from flag-motion-based aerodynamic improvements [1] to the design of cooling systems [33] and musical instruments [3]. Recently, interest in this canonical FSI problem has been sustained with the emergence of innovative energy harvesting concepts [21,37] and new needs for the understanding of biomechanical systems, such as flutter of the soft-palate in the upper airway during snoring $[4,7,38]$. For many immersed-cantilever systems, the large scale of the structures and/or the high flow speeds encountered give rise to high-Reynolds-number environments, meaning that inertial effects of the flow predominate and the explicit effects of viscosity can be neglected. However, for small- and micro-scale systems in the fields of energy harvesting and biomechanics, the FSI at low flow speeds is of interest. In such cases, the effect of viscosity on immersed-cantilever stability requires more detailed examination.

Until now, most studies of the FSI system under consideration have adopted linear structural mechanics using the one-dimensional Euler-Bernoulli beam equation. They have also assumed infinitely high Reynolds numbers and

\footnotetext{
*Corresponding author

Email address: Julien.Cisonni@curtin.edu.au (Julien Cisonni)
} 


\begin{tabular}{|c|c|c|c|}
\hline \multicolumn{2}{|c|}{ Nomenclature } & $\mathbf{R}_{c}^{*}$ & position vector to a material point in the deformed can- \\
\hline$B^{*}$ & flexural rigidity of flexible cantilever & & tilever centreline ( 1 -D beam model) \\
\hline $\mathrm{Ca}$ & Cauchy number & $\operatorname{Re}$ & Reynolds number \\
\hline$C a_{\text {eff }}$ & effective Cauchy number & $U$ & reduced (mean inlet) velocity \\
\hline$E^{* 11}$ & Young's modulus of flexible cantilever & $U^{*} u^{*}$ & mean inlet velocity \\
\hline $\begin{array}{l}E_{\mathrm{eff}}^{*} \\
f^{*}\end{array}$ & $\begin{array}{l}\text { 1-D effective Young's modulus of flexible cantilever } \\
\text { frequency }\end{array}$ & $u_{1}, u_{2}$ & {$\left[\mathbf{u}^{*}=\left\langle u_{1}^{*}, u_{2}^{*}\right\rangle\right]$} \\
\hline$f_{C}^{*}$ & characteristic frequency of flexible cantilever & $U_{\text {crit }}^{*}$ & critical (mean inlet) velocity \\
\hline$f_{\text {crit }}^{*}$ & critical oscillation frequency of flexible cantilever & $t^{*}$ & time \\
\hline$f_{n}^{*}$ & $\begin{array}{l}n^{\text {th }} \text { eigenfrequency of flexible cantilever (in vacuo linear } \\
\text { theory) }\end{array}$ & $\mathbf{T}^{*}$ & $\begin{array}{l}\text { load applied on flexible-cantilever boundaries ( } 2 \text {-D solid } \\
\text { model) }\end{array}$ \\
\hline $\begin{array}{l}f_{\mathrm{T}}^{*} \\
H^{*}\end{array}$ & $\begin{array}{l}\text { frequency of cantilever tip oscillations } \\
\text { height of channel }\end{array}$ & $\mathbf{T}_{\mathrm{eff}}^{*}$ & $\begin{array}{l}\text { load combining the tractions acting at the top and bottom } \\
\text { of the cantilever ( } 1 \text {-D beam model) }\end{array}$ \\
\hline$h_{\mathrm{C}}^{*}$ & thickness of flexible cantilever & $x_{1}^{*}, x_{2}^{*}$ & axial and transverse Eulerian coordinates $\left[\mathbf{x}^{*}=\left\langle x_{1}^{*}, x_{2}^{*}\right\rangle\right]$ \\
\hline $\begin{array}{l}h_{L} \\
L^{*}\end{array}$ & $\begin{array}{l}\text { cantilever thickness-to-length ratio } \\
\text { length of channel }\end{array}$ & $\alpha_{\mathrm{T}}^{*}$ & exponential growth/decay rate of cantilever tip oscilla- \\
\hline$L_{\mathrm{C}}^{*}$ & length of flexible cantilever & & $\begin{array}{l}\text { tions } \\
\text { transverse deflection of flexible cantilever }\end{array}$ \\
\hline$L_{H}$ & cantilever-length-to-channel-height ratio & $\eta_{\mathrm{T}}^{*}$ & transverse deflection of flexible-cantilever tip \\
\hline$L_{\text {inlet }}^{*}$ & length of inlet-dividing rigid wall & $\eta_{\mathrm{T} 0}^{*}$ & initial transverse deflection of flexible-cantilever tip \\
\hline$M^{\text {outl }}$ & fluid-to-solid mass ratio & $\mu_{\mathrm{F}}^{*}$ & dynamic viscosity of fluid \\
\hline$p^{*}$ & fluid pressure & & Poisson's ratio of flexible cantilever \\
\hline $\mathbf{r}^{*}$ & $\begin{array}{l}\text { position vector to a material point in the undeformed } \\
\text { cantilever ( } 2 \text {-D solid model) }\end{array}$ & $\xi_{\xi_{1}^{*}}^{*}, \xi_{2}^{*}$ & $\begin{array}{l}\text { axial Lagrangian coordinate (1-D beam model) } \\
\text { axial and transverse Lagrangian coordinates }(2-D \text { solid }\end{array}$ \\
\hline $\mathbf{R}^{*}$ & $\begin{array}{l}\text { position vector to a material point in the deformed can- } \\
\text { tilever (2-D solid model) }\end{array}$ & $\rho_{\mathrm{C}}^{*}$ & $\begin{array}{l}\text { model) }\left[\boldsymbol{\xi}^{*}=\left\langle\xi_{1}^{*}, \xi_{2}^{*}\right\rangle\right]_{\text {density of flexible cantilever }} \\
\text { den }\end{array}$ \\
\hline $\mathbf{r}_{c}^{*}$ & $\begin{array}{l}\text { position vector to a material point in the undeformed } \\
\text { cantilever centreline (1-D beam model) }\end{array}$ & $\rho_{\mathrm{F}}^{*}$ & $\begin{array}{l}\text { density of fluid } \\
\text { dimensional parameters }\end{array}$ \\
\hline
\end{tabular}

employed ideal flow models with an imposed Kutta condition [18, 22, 23] that implicitly models the effect of viscosity. Thus, immersed flexible cantilevers have been shown to lose their stability to a single-mode flutter instability at a critical value of flow speed dependent of the fluid-to-solid mass ratio. The destabilisation mechanism is fundamentally due to an irreversible energy transfer from fluid to structure. This arises from a phase difference between fluid pressure and cantilever motion that owes its origin to the finite length of the flexible cantilever [15, 22]. More recent analysis and modelling efforts have particularly focused on the wake downstream of the fluttering cantilever $[28,34,36]$ and three-dimensional effects [11, 17, 24]. However, viscous effects most often remain approximated [27] or implicitly modelled [2].

As the higher computational cost can be significant, relatively few investigators $[4,9,33,38,41]$ have employed the Navier-Stokes equations to explicitly take into account fluid viscosity in the simulation of the motion of immersed cantilevers. In general, these numerical investigations have aimed to obtain more detailed predictions of the flow field and the vortex dynamics downstream of the cantilever free end. Shoele and Mittal [33] have shown that a more detailed characterisation of the downstream flow can be particularly important for the optimisation of the convective heat-transfer performance of cantilever-based thermal systems for the laminar channel-flow regime $(R e<1500)$. However, most studies based on the solution of the Navier-Stokes equations only considered moderate Reynolds numbers $\left(R e \sim 10^{2}\right.$ ) or were restricted to particular system configurations, for instance in biomedical applications such as snoring $[4,38]$. Thus, to date, only limited work on the effects of viscosity on the stability of a channelimmersed cantilever has been conducted.

The present study comprises a comprehensive parametric analysis of the FSI dynamics of a flexible cantilever immersed in viscous channel flow. Its purpose is to extend to the viscous case the detailed investigations conducted by, among others, Guo and Païdoussis [18] and Howell et al. [22] on the flutter instability of flexible cantilevers immersed in inviscid axial flow. The effect of slenderness of the flexible cantilever on its motion is first analysed by comparing the predictions of geometrically linear and non-linear elastic beam models with those of a 2-D solid model. Time-marching simulations based on the coupling of a one-dimensional elastic cantilever, allowing for geometric nonlinearity, and the Navier-Stokes equations are then used to explore the characteristics of the cantilever motion and its instability boundaries for finite Reynolds numbers $R e<1000$, based on the channel height. The dependence of the flutter-instability characteristics, pre- and post-critical, on the Reynolds number and the cantilever confinement is investigated at fluid-to-solid mass ratios ranging from 0.1 to 10 . Further results illustrate the variations of predicted critical velocity and frequency values associated with the changes in the balance between viscous and inertial forces 

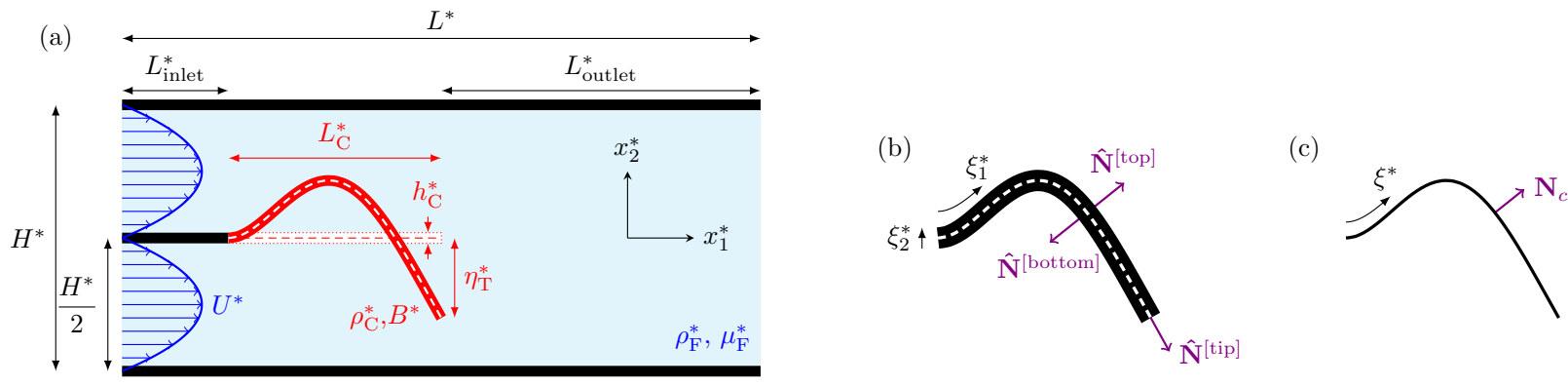

Figure 1: Description of the FSI system modelling a flexible cantilever immersed in a viscous channel flow, indicating (a) the physical quantities of the problem and the Lagrangian coordinates of the (b) two-dimensional solid model and (c) one-dimensional beam model.

within the fluid flow.

\section{Method}

The FSI system studied is a flexible cantilever of length $L_{\mathrm{C}}^{*}$ and thickness $h_{\mathrm{C}}^{*}$ immersed in a viscous fluid flowing in a two-dimensional channel of length $L^{*}$ and height $H^{*}[7,8,12,13]$. The fluid is characterised by its density $\rho_{\mathrm{F}}^{*}$ and dynamic viscosity $\mu_{\mathrm{F}}^{*}$ while the cantilever is characterised by its density $\rho_{\mathrm{C}}^{*}$ and flexural rigidity $B^{*}=E^{*} h_{\mathrm{C}}^{* 3} /(12(1-$ $\left.v^{2}\right)$ ), where $E^{*}$ and $v=0.4$ are, respectively, Young's modulus and the Poisson ratio of the cantilever material. The flexible cantilever is positioned along the centreline of the channel and parallel to the channel walls. It is clamped to a rigid wall of length $L_{\text {inlet }}^{*}$ dividing the upstream end of the channel into two inlets of identical height $H^{*} / 2$. Identical steady Poiseuille flows having an average velocity $U^{*}$ are imposed at both inlets. At the channel outlet, located at a distance $L_{\text {outlet }}^{*}$ from the downstream free end of the flexible cantilever, the flow is assumed to be parallel and axially traction-free. A schematic of the system configuration is shown in Fig. 1(a). Throughout this paper, all dimensional quantities are identified by an asterisk.

\subsection{System parameterisation}

The FSI system is analysed in non-dimensional form by scaling the geometric dimensions and spatial coordinates with the channel height $H^{*}$, the flow velocity components with the average inlet velocity $U^{*}$, the fluid pressure with the dynamic pressure $\rho_{\mathrm{F}}^{*} U^{* 2}$, the solid stresses and loads with Young's modulus $E^{*}$ (or, for the beam models, effective Young's modulus $E_{\mathrm{eff}}^{*}=E^{*} /\left(1-v^{2}\right)$ ), and time with $H^{*} / U^{*}$. Using this non-dimensionalisation scheme, the dynamics of the immersed cantilever are characterised by five non-dimensional parameters defined as

$$
M=\frac{\rho_{\mathrm{F}}^{*} L_{\mathrm{C}}^{*}}{\rho_{\mathrm{C}}^{*} h_{\mathrm{C}}^{*}}, \quad U=U^{*} L_{\mathrm{C}}^{*} \sqrt{\frac{\rho_{\mathrm{C}}^{*} h_{\mathrm{C}}^{*}}{B^{*}}}, \quad L_{H}=\frac{L_{\mathrm{C}}^{*}}{H^{*}}, \quad R e=\frac{\rho_{\mathrm{F}}^{*} U^{*} H^{*}}{\mu_{\mathrm{F}}^{*}}, \quad h_{L}=\frac{h_{\mathrm{C}}^{*}}{L_{\mathrm{C}}^{*}} .
$$

The mass ratio $M$ is the ratio between the fluid and structural inertias. The reduced velocity $U$, which can be interpreted as the inverse of the FSI Strouhal number, is the ratio between the fluid convective timescale and that of the characteristic in vacuo vibration of the flexible structure. Both $M$ and $U$ are the primary parameters determining the stability of any flexible structure interacting with a surrounding flow. In the present configuration, for which the cantilever is immersed in a viscous channel flow, the confinement of the flexible structure is accounted for by the ratio $L_{H}$ between cantilever-length and channel-height, and viscous effects by the Reynolds number Re. The cantilever thickness-to-length ratio $h_{L}$ measures the slenderness of the flexible structure.

\subsection{Problem formulation}

The fluid motion in the Eulerian coordinate system $\mathbf{x}=\left\langle x_{1}, x_{2}\right\rangle$, of which the unit vectors are $\mathbf{e}_{1}$ and $\mathbf{e}_{2}$, is governed by the incompressible Navier-Stokes equations

$$
\frac{\partial \mathbf{u}}{\partial t}+\mathbf{u} \cdot \nabla \mathbf{u}=-\nabla p+\frac{1}{R e} \nabla^{2} \mathbf{u}
$$


and the continuity equation

$$
\nabla \cdot \mathbf{u}=0
$$

where $\mathbf{u}$ and $p$ are, respectively, the non-dimensional fluid velocity and pressure.

The flexible cantilever is first modelled as a two-dimensional linearly elastic Hookean solid (later referred to as the 2-D solid model). Its shape is parameterised by the Lagrangian coordinates $\boldsymbol{\xi}=\left\langle\xi_{1}, \xi_{2}\right\rangle$, shown in Fig. 1(b), so that the position vector to a material point in the undeformed cantilever is given by $\mathbf{r}(\boldsymbol{\xi})$. The deformation of the flexible cantilever, subjected to a load $\mathbf{T}$ applied on its boundaries, is then described by the vector field that specifies the position vectors to material points in the deformed configuration given by $\mathbf{R}(\boldsymbol{\xi})$. The principle of virtual displacements (PVD) that governs the cantilever motion is given by

$$
\int_{S_{\mathrm{C}}}\left\{\boldsymbol{\sigma}: \delta \boldsymbol{\gamma}-\left(\mathbf{f}-\frac{C a}{M h_{L}} \frac{\partial^{2} \mathbf{R}}{\partial t^{2}}\right) \cdot \delta \mathbf{R}\right\} d s-\oint_{D_{\mathrm{C}}}\{\mathbf{T} \cdot \delta \mathbf{R}\} d D=0,
$$

where

$$
C a=\frac{\rho_{\mathrm{F}}^{*} U^{* 2}}{E^{*}}=\frac{M U^{2} h_{L}^{3}}{12\left(1-v^{2}\right)}
$$

is the Cauchy number representing the ratio between the fluid inertial forces and the solid elastic forces, $\sigma$ is the symmetric second Piola-Kirchhoff stress tensor, $\delta \gamma$ is the variation of the Green strain tensor, $\mathbf{f}$ is the body force per unit area of the undeformed solid, $S_{\mathrm{C}}$ is the reference surface of the undeformed solid and $D_{\mathrm{C}}$ is the fluid-solid interface on which the load $\mathbf{T}$ is applied. Linearly elastic, compressible behaviour is assumed so that

$$
\sigma=\frac{1}{(1+v)}\left(\frac{v}{(1-2 v)} \operatorname{tr}(\boldsymbol{\gamma}) \mathbf{I}+\boldsymbol{\gamma}\right)
$$

where $v<0.5$.

When the cantilever is sufficiently slender, typically for a cantilever thickness-to-length ratio $h_{L}<1 / 20$, the elastic solid can be described by one-dimensional beam theory. The shape of the flexible cantilever is then assumed to be characterised by the solid centreline and parameterised by the Lagrangian coordinate $\xi$, shown in Fig. 1(c), so that the position vector to a material point in the undeformed cantilever centreline is given by $\mathbf{r}_{c}(\xi)$. The flexible cantilever can be modelled as a one-dimensional elastic Kirchhoff-Love beam (later referred to as the 1-D beam model), allowing for geometric non-linearity. Its deformation, due to a resultant load $\mathbf{T}_{\text {eff }}$ combining the tractions acting at the top and bottom of the beam, results in a displacement of the material points to their new positions $\mathbf{R}_{c}(\xi)$, which are determined by the PVD

$$
\int_{0}^{L_{H}}\left\{\gamma \delta \gamma+\frac{h_{L}^{2} L_{H}^{2}}{12} \kappa \delta \kappa-\left(\frac{1}{h_{L} L_{H}} \sqrt{\frac{A}{a}} \mathbf{T}_{\mathrm{eff}}-\frac{C a_{\mathrm{eff}}}{M h_{L}} \frac{\partial^{2} \mathbf{R}_{c}}{\partial t^{2}}\right) \cdot \delta \mathbf{R}_{c}\right\} \sqrt{a} d \xi=0
$$

where

$$
C a_{\mathrm{eff}}=\frac{\rho_{\mathrm{F}}^{*} U^{* 2}}{E_{\mathrm{eff}}^{*}}=\left(1-v^{2}\right) \frac{\rho_{\mathrm{F}}^{*} U^{* 2}}{E^{*}}=\left(1-v^{2}\right) C a=\frac{M U^{2} h_{L}^{3}}{12}
$$

is the effective Cauchy number, and

$$
a=\frac{\partial \mathbf{r}_{c}}{\partial \xi} \cdot \frac{\partial \mathbf{r}_{c}}{\partial \xi}, \quad A=\frac{\partial \mathbf{R}_{c}}{\partial \xi} \cdot \frac{\partial \mathbf{R}_{c}}{\partial \xi}
$$

are the squares of the lengths of infinitesimal material line elements in the undeformed and deformed configurations, respectively. Therefore, the ratio $\sqrt{A / a}$ represents the stretch of the cantilever centreline while the strain $\gamma$ and bending $\kappa$ are given by

$$
\gamma=\frac{1}{2}(A-a), \quad \kappa=-(C-c)
$$

with

$$
c=\mathbf{n}_{c} \cdot \frac{\partial^{2} \mathbf{r}_{c}}{\partial \xi^{2}}, \quad C=\mathbf{N}_{c} \cdot \frac{\partial^{2} \mathbf{R}_{c}}{\partial \xi^{2}}
$$


representing the curvature of the cantilever centreline, respectively, before and after the deformation. $\mathbf{n}_{c}$ and $\mathbf{N}_{c}$ denote the unit normals (pointing into the fluid) to the top face of the undeformed and deformed cantilever centreline, respectively.

When the flexible cantilever is immersed in the channel flow, the traction $\mathbf{T}_{\text {fluid }}$ exerted by the flowing fluid on the solid boundaries depends on the pressure and viscous shear stress, so that

$$
\mathbf{T}=\mathbf{T}_{\text {fluid }}=C a\left\{\left(p \mathbf{I}-\frac{1}{R e}\left(\nabla \mathbf{u}+(\nabla \mathbf{u})^{\mathrm{T}}\right)\right) \cdot \hat{\mathbf{N}}\right\},
$$

where $\hat{\mathbf{N}}$ denotes the unit normal (pointing into the fluid) to the boundary on which the traction is applied. In the 1-D beam model, the fluid load that acts on the cantilever centreline, combining the fluid tractions acting on its top and bottom faces, can be expressed as

$$
\mathbf{T}_{\text {eff }}=C a_{\text {eff }}\left\{\left(\left.p\right|_{\text {top }} \mathbf{I}-\left.\frac{1}{R e}\left(\nabla \mathbf{u}+(\nabla \mathbf{u})^{\mathrm{T}}\right)\right|_{\text {top }}\right) \cdot \mathbf{N}_{c}-\left(\left.p\right|_{\text {bottom }} \mathbf{I}-\left.\frac{1}{R e}\left(\nabla \mathbf{u}+(\nabla \mathbf{u})^{\mathrm{T}}\right)\right|_{\text {bottom }}\right) \cdot \mathbf{N}_{c}\right\}
$$

For small deformations, the motion of the cantilever can be assumed to be characterised by the vertical deflection $\eta\left(x_{1}\right)$ of the solid centreline in the Eulerian coordinate system. Also, the beam becomes approximately inextensible and the bending becomes purely dependent on the transverse deflection. Furthermore, the viscous normal stresses acting on the cantilever vanish, as for an ideal flow. Therefore the combined fluid traction that acts on the solid centreline is given by the distributed pressure difference $\Delta p\left(x_{1}\right)$, between the top and bottom faces of the cantilever, so that $\mathbf{T}_{\text {eff }}=\Delta p \mathbf{e}_{2}$. The motion of the cantilever is then governed by the Euler-Bernoulli beam equation

$$
\frac{\partial^{2} \eta}{\partial t^{2}}+\frac{L_{H}^{2}}{U^{2}} \frac{\partial^{4} \eta}{\partial x_{1}^{4}}=\frac{M}{L_{H}} \Delta p
$$

This simplified formulation of the cantilever dynamics, used in numerous studies [11, 18, 21, 22], provides a useful reference to the in vacuo analytical solution (i.e. $\Delta p=0$ ).

\subsection{Boundary and initial conditions}

The flexible cantilever is free at its downstream end and is clamped to a rigid wall at the upstream end. In the 1-D beam model, the clamped boundary condition is given by

$$
\mathbf{R}_{c}(\xi=0)=\mathbf{r}_{c}(\xi=0),\left.\quad \frac{\mathrm{d}\left(\mathbf{R}_{c} \cdot \mathbf{e}_{2}\right)}{\mathrm{d} \xi}\right|_{\xi=0}=0 .
$$

At both inlets, a parabolic velocity profile is imposed for the axial velocity so that

$$
\mathbf{u}= \begin{cases}12 x_{2}\left(1-2 x_{2}\right) \mathbf{e}_{1}, & 0 \leq x_{2} \leq H / 2 \text { (upper inlet) } \\ -12 x_{2}\left(1+2 x_{2}\right) \mathbf{e}_{1}, & -H / 2 \leq x_{2} \leq 0 \text { (lower inlet) }\end{cases}
$$

At the outlet, the transverse velocity is set to zero to ensure a parallel flow and the axially-traction-free outflow condition is used to determine the axial velocity. The no-slip condition given by $\mathbf{u}=0$ is applied on the stationary walls. On the flexible-cantilever walls, the no-slip condition is given by

$$
\mathbf{u}=\frac{\partial \hat{\mathbf{R}}}{\partial t}
$$

where $\hat{\mathbf{R}}$ is the position vector of the FSI interface.

The time-dependent simulations were started from an initial condition in which the cantilever was deformed approximately into the $n^{\text {th }}$ in vacuo mode shape of the linear Euler-Bernoulli beam,

$$
\eta_{n}\left(x_{1}\right)=\frac{\eta_{\mathrm{T}}}{2}\left[\left(\cosh \left(\beta_{n} \frac{x_{1}}{L_{\mathrm{C}}}\right)-\cos \left(\beta_{n} \frac{x_{1}}{L_{\mathrm{C}}}\right)\right)-\frac{C_{n}}{S_{n}}\left(\sinh \left(\beta_{n} \frac{x_{1}}{L_{\mathrm{C}}}\right)-\sin \left(\beta_{n} \frac{x_{1}}{L_{\mathrm{C}}}\right)\right)\right],
$$


where $\eta_{\mathrm{T}}$ is the deflection amplitude at the free-end tip, $C_{n}=\cos \left(\beta_{n}\right)+\cosh \left(\beta_{n}\right), S_{n}=\sin \left(\beta_{n}\right)+\sinh \left(\beta_{n}\right)$, and $\beta_{n}$ satisfies the dispersion relation $\cos \left(\beta_{n}\right) \cosh \left(\beta_{n}\right)=-1$ [29]. This deflection was imposed by determining the beam's steady state deformation in response to the traction

$$
\mathbf{T}_{\text {eff }}=\frac{h_{\mathrm{C}}^{3}}{12} \frac{d^{4} \eta_{n}\left(x_{1}\right)}{d x_{1}^{4}} \mathbf{e}_{2} .
$$

For the 2-D solid model the initial shape of the cantilever was determined by subjecting its top face to the equivalent load

$$
\mathbf{T}=\frac{h_{\mathrm{C}}^{3}}{12\left(1-v^{2}\right)} \frac{d^{4} \eta_{n}\left(x_{1}\right)}{d x_{1}^{4}} \mathbf{e}_{2} .
$$

For the linear beam model, the traction (19) deforms the beam exactly into the in vacuo mode shape (18). For the non-linear beam model and the 2-D solid model, the applied load produces a slightly different deflection (see Section 3 for a detailed comparison between the three solid models).

It is known from previous studies (e.g. [30]) that for fluid-immersed clamped-free cantilevers, only structural modes of order $n \geq 2$ become unstable. Therefore, most simulations were performed with an initial condition corresponding to Mode $2\left(\beta_{2}=4.6941\right)$. The influence of alternative initial mode shapes, such as Mode $1\left(\beta_{1}=1.8751\right)$ and Mode $3\left(\beta_{3}=7.8548\right)$, on the static and dynamic responses of the flexible cantilever is discussed in Section 3 for the in vacuo case and in Section 5 for the immersed case.

\subsection{Discretisation and implementation}

The problem is implemented using the open-source finite-element library oomph-lib [19], allowing the FSI system to be solved numerically with either the 1-D beam or 2-D solid model.

In the 1-D beam model, the cantilever is spatially discretised using two-node Hermite finite elements whereas in the 2-D solid model the flexible structure is spatially discretised using nine-node quadrilateral displacement-based solid mechanics finite elements. The Navier-Stokes equations are discretised on a moving, body-fitted mesh, using nine-node quadrilateral Taylor-Hood elements. The fluid mesh update is performed by an algebraic node update procedure, based on a generalisation of Kistler and Scriven's [25] "method of splines". Details of this approach and its implementation can be found in the extensive tutorials available on the oomph-lib webpages [19]. Both solid and fluid nine-node elements are implemented with adaptive mesh refinement capabilities.

Time stepping is implemented using a Newmark scheme for the solid and a second-order backward differentiation formula scheme for the fluid; the relevant mesh velocity terms are included in the evaluation of the Eulerian timederivatives. The FSI problem is discretised monolithically and the Newton-Raphson method is used to solve the non-linear system of equations, Eqs. (2), (3) and (4) for the immersed 2-D solid model or Eqs. (2), (3) and (7) for the immersed 1-D beam model, employing the SuperLU direct linear solver within the Newton iteration. While the use of a monolithic discretisation guarantees maximum robustness of the computational procedure, it is possible that in certain weakly-coupled cases a segregated approach may have been more efficient. This issue is not explored in the current study but a detailed comparison of the relative merits of the two approaches has been performed by Heil et al. [20].

\subsection{Numerical simulations}

The stability analysis of the FSI system is carried out for variations of the four main non-dimensional parameters, $M, U, L_{H}$ and $R e$, as shown in Table 1. The fifth parameter, $h_{L}$, characterising the cantilever-section aspect ratio, varies with $L_{H}$ but only within a range of values for which the results are not significantly affected, as discussed in Section 3. The main analysis follows an approach similar to previous investigations [33, 35, 41] and consists in running time-marching simulations of the FSI system, containing the 1-D beam model, for all combinations of $(M, U)$ and $\left(L_{H}, R e\right)$ pairings. Therefore, the properties of the flexible-cantilever oscillations can be analysed over the whole $(M, U)$ domain considered, similar to studies based on inviscid FSI models [15, 18, 22], for different combinations of $\left(L_{H}, R e\right)$. A total of 121,730 simulations were run, consuming approximately 150,000 CPU hours on Intel Xeon Westmere-EP X5660 $2.8 \mathrm{GHz}$ processors. 
Table 1: Non-dimensional parameter values used for the FSI simulations.

\begin{tabular}{lll}
\hline Parameter & \multicolumn{2}{c}{ Value(s) } \\
\hline Mass ratio & $M$ & 37 values logarithmically spaced between 0.1 and 10 \\
Reduced velocity & $U$ & 47 values logarithmically spaced between 0.2 and 100 \\
Cantilever-length-to-channel-height ratio & $L_{H}$ & $\{1,2,3,4,5,8,10\}$ \\
Reynolds number & $R e$ & $\{1,2,5,10,20,50,100,200,500,1000\}$ \\
\hline Cantilever thickness-to-length ratio & $h_{L}$ & $1 /\left(50 L_{H}\right)\left(\right.$ note: $\left.h_{\mathrm{C}}=h_{\mathrm{C}}^{*} / H^{*}=1 / 50\right)$ \\
Initial tip deflection amplitude & $\eta_{\mathrm{T} 0}$ & $h_{\mathrm{C}} / 4$ \\
\hline Inlet splitter wall length & $L_{\text {inlet }}$ & 1 \\
Distance from cantilever free end to outlet & $L_{\text {outlet }}$ & $2\left(L_{\text {inlet }}+L_{H}\right)$ \\
Channel total length & $L$ & $L_{\text {inlet }}+L_{H}+L_{\text {outlet }}$ \\
\hline
\end{tabular}

For each run corresponding to a set of non-dimensional parameters $\left(L_{H}, R e, M, U\right)$, the start-up procedure involves (I) applying on the top face of the flexible cantilever the external traction defined in Eq. (19) (Eq. (20) in the 2-D solid model) under no-flow conditions to deflect it into a Mode 2 shape with an approximated amplitude $\eta_{\mathrm{T} 0}$ (see Table 1 and Section 3 for discussion) at the tip, (II) constraining the cantilever in position, (III) gradually introducing the flow in a sequence of steady solutions then (IV) releasing the cantilever and solving the unsteady FSI problem. In each computation, at least 10 periods of the flexible-cantilever oscillation were simulated with at least 50 time steps per period. Over a particular range of non-dimensional parameters, however, the FSI system is strongly unstable so the cantilever has a rapidly growing deflection and hits the channel walls in fewer than 10 periods. As the problem formulation does not include a model of contact mechanics, the simulations for such cases were stopped before their target number of time steps, resulting in truncated analysable data.

The adaptive mesh refinement remained active throughout the simulations and all elements were allowed to be refined or coarsened once per time step. Preliminary simulations were conducted to verify that the time step size $\Delta t$, the number of elements used for the spatial discretisation of the flexible cantilever, the adaptive mesh refinement parameters and the tolerance in the iterative processes were chosen appropriately to ensure satisfactory convergence of the numerical model's predictions. Alternative initial start-up procedures were tested to ensure that the final results used to characterise the FSI system stability were independent of this input.

\subsection{Stability characterisation}

For each set of non-dimensional parameters $\left(L_{H}, R e, M, U\right)$, the FSI system was characterised by the time trace of the deflection of the flexible-cantilever tip $\eta_{\mathrm{T}}$, or the flexible-cantilever centreline tip for the 2-D model. The oscillation of the cantilever tip was assumed to be of the dimensional form

$$
\eta_{\mathrm{T}}^{*}=\eta_{\mathrm{T}}^{\dagger *} e^{\alpha_{\mathrm{T}}^{*} t^{*}} \cos \left(2 \pi f_{\mathrm{T}}^{*} t^{*}+\phi^{\dagger}\right)
$$

where $\eta_{\mathrm{T}}^{\dagger *}$ is a reference initial amplitude, and $\alpha_{\mathrm{T}}^{*}, f_{\mathrm{T}}^{*}$ and $\phi^{\dagger}$, respectively, the exponential growth rate, the frequency and the phase of the oscillation. The stability or instability of the system was then determined from the exponential growth rate normalised by the tip oscillation frequency $\alpha_{\mathrm{T}}=\alpha_{\mathrm{T}}^{*} / f_{\mathrm{T}}^{*}$. The tip oscillation frequency, like all the other frequencies, was non-dimensionalised on the characteristic in vacuo frequency of the flexible cantilever $f_{\mathrm{C}}^{*}=\sqrt{B^{*} /\left(\rho_{\mathrm{C}}^{*} h_{\mathrm{C}}^{*}\right)} / L_{\mathrm{C}}^{* 2}$, so that $f_{\mathrm{T}}=f_{\mathrm{T}}^{*} / f_{\mathrm{C}}^{*}$. Figure 2 illustrates the stability analysis procedure in which $\alpha_{\mathrm{T}}$ is estimated through a linear interpolation of the log envelope detected on the signal $\eta_{\mathrm{T}}$, as shown in Fig. 2(a), and $f_{\mathrm{T}}$ is estimated through a peak detection on a 4096-FFT of the signal $\eta_{\mathrm{T}}$, as shown in Fig. 2(b) for $\left(L_{H}=2, \operatorname{Re}=100, M=3\right)$ and $U=1,5$ and 17.1 .

When the flexible cantilever oscillates in a single mode shape (e.g. for $U=1$ and 17.1), the signal form assumed in Eq. (21) is appropriate. Thus, only one main frequency component is observed in the FFT and the linear interpolation of the log envelope gives an accurate estimation of the decay or growth of the oscillations. However, for particular sets of parameters (e.g. $U=5$ ), the flexible-cantilever motion can be dominated by a combination of two modes. For such cases, the signal $\eta_{\mathrm{T}}$ is actually the sum of two exponentially decaying/growing cosine waves and the form assumed in Eq. (21) becomes inadequate. Therefore, in the vicinity of modal-transition regions in the parameter space, the estimations of the growth rate $\alpha_{\mathrm{T}}$ and the frequency $f_{\mathrm{T}}$ are determined with a greater uncertainty. Nonetheless, this 

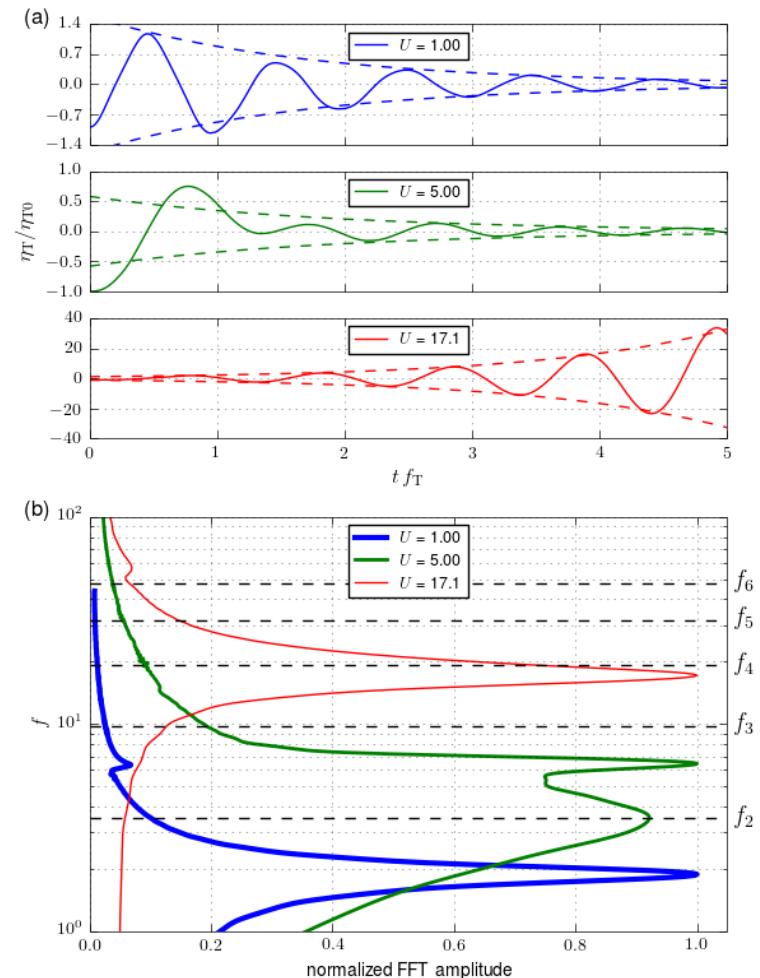
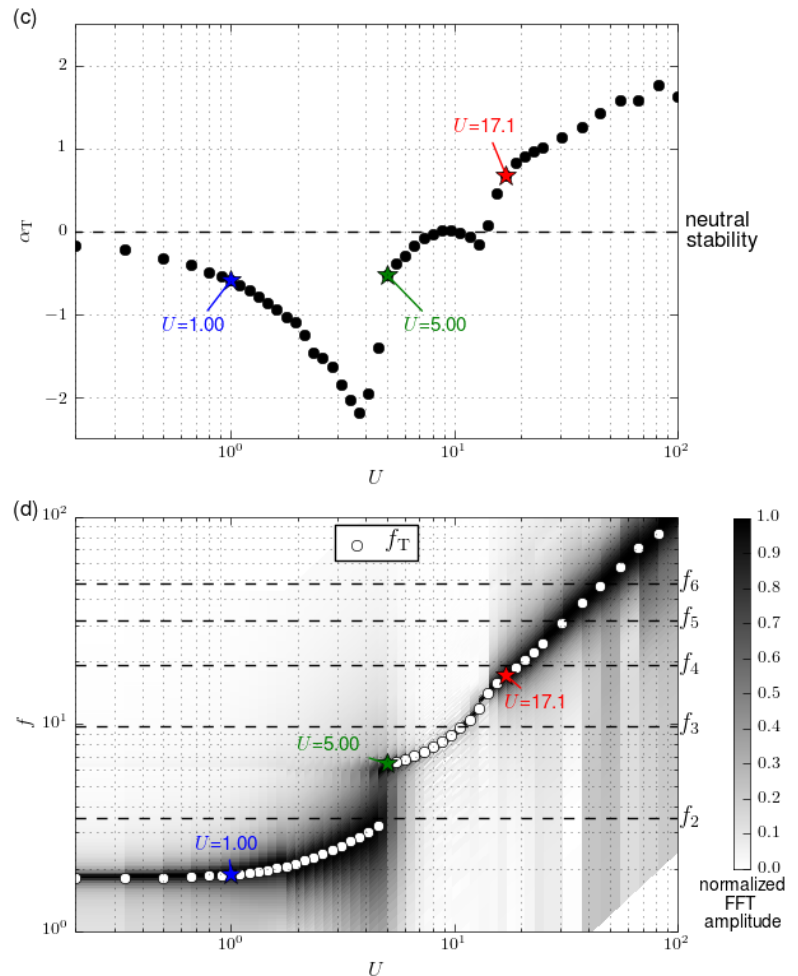

Figure 2: Illustration of the stability analysis of the FSI system containing the 1-D beam model from the numerical simulations for $L_{H}=2, R e=100$ and $M=3$ : (a) Time trace of the flexible-cantilever tip deflection (solid line) for $U=1.0,5.0$ and 17.1, and associated estimated envelope (dashed line). (b) FFT of the time traces shown in (a). (c) Exponential growth rate $\alpha_{\mathrm{T}}$ and (d) FFT of the time trace of the flexible-cantilever tip deflection as functions of the reduced velocity $U$. The cantilever oscillation frequency $f_{\mathrm{T}}$ estimated from the FFT is indicated with round markers in (d). The cantilever eigenfrequencies $f_{n}$ (in vacuo linear theory) associated with Modes 2 to 6 are indicated with dashed lines in (b) and (d).

only arises for relatively few cases and does not significantly affect the coherence of the system characterisation in the whole parameter space. Thus, for instance, the evolution of $\alpha_{\mathrm{T}}$ and $f_{\mathrm{T}}$ as functions of $U$, as shown in Fig. 2(c) and (d), is consistent with results obtained experimentally [40].

\section{Cantilever in vacuo characterisation}

A preliminary analysis of the steady and unsteady motions of the flexible cantilever subject to an initial external load is carried out for the in vacuo case. This study aims to characterise the static and dynamic responses of the structural part of the FSI system predicted with both 1-D beam and 2-D solid models, and how these predicted responses relate to each other and to linear Euler-Bernoulli theory. It focuses on two major aspects of the cantilever modelling: the slender beam approximation, through variations of the cantilever thickness-to-length ratio $h_{L}$, and the geometric non-linearity associated with large displacements, through variations of the ratio of initial deflection of the cantilever tip $\eta_{\mathrm{T} 0}$ to cantilever length $L_{\mathrm{C}}$.

For the in vасио case, the geometric dimensions and spatial coordinates are scaled with the cantilever length $L_{\mathrm{C}}^{*}$ and time with the characteristic intrinsic timescale of the solid $L_{\mathrm{C}}^{*} \sqrt{\rho_{\mathrm{C}}^{*} / E^{*}}$ or $L_{\mathrm{C}}^{*} \sqrt{\rho_{\mathrm{C}}^{*} / E_{\mathrm{eff}}^{*}}$. Because of the change in nondimensionalisation, all the non-dimensional FSI parameters in the solid equations lose their significance. Therefore, the ratios $\mathrm{Ca} /\left(M h_{L}\right)$ and $C a_{\text {eff }} /\left(M h_{L}\right)$ in Eqs. (4) and (7), respectively, and the cantilever-length-to-channel-height ratio $L_{H}$ are set to the default value of unity.

First, the structural static response is characterised as a function of the two in vacuo parameters $h_{L}$ and $\eta_{\mathrm{T} 0} / L_{\mathrm{C}}$ for the $1^{\text {st }}, 2^{\text {nd }}$ and $3^{\text {rd }}$ modes by the transverse displacements of the cantilever tip when an external traction, of the form given in Eqs. (20) or (19) for Mode 2, is applied to produce an initial small-amplitude (linear regime) modal 

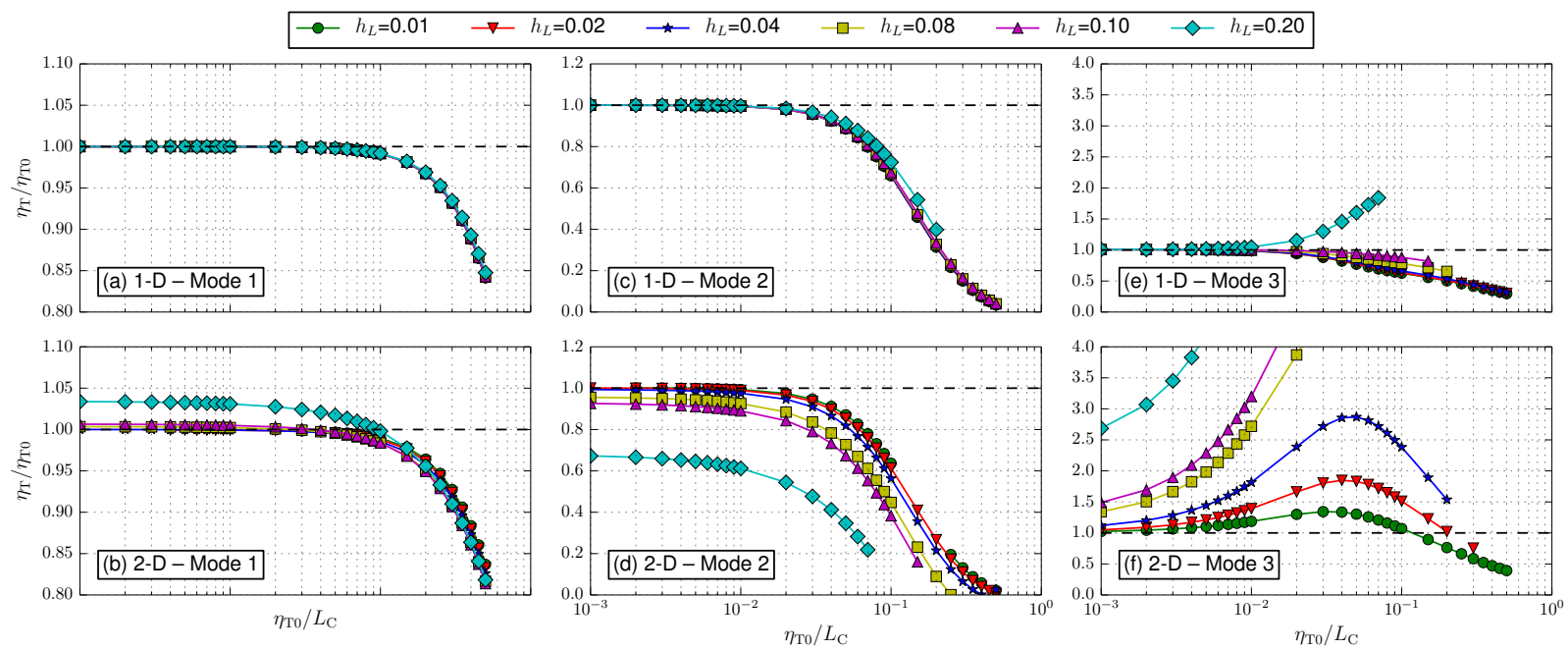

Figure 3: Analysis of the in vacuo static response of the 1-D beam (top) and 2-D solid (bottom) models: Convergence of the deflection amplitude at the tip $\eta_{\mathrm{T}}$ to the linear theory as a function of the ratio between eigenmode deflection amplitude at the tip $\eta_{\mathrm{T} 0}$ and cantilever length $L_{\mathrm{C}}$ for several cantilever thickness-to-length ratios $h_{L}$, and Mode 1 (a,b), Mode 2 (c,d) and Mode 3 (e,f) shapes.

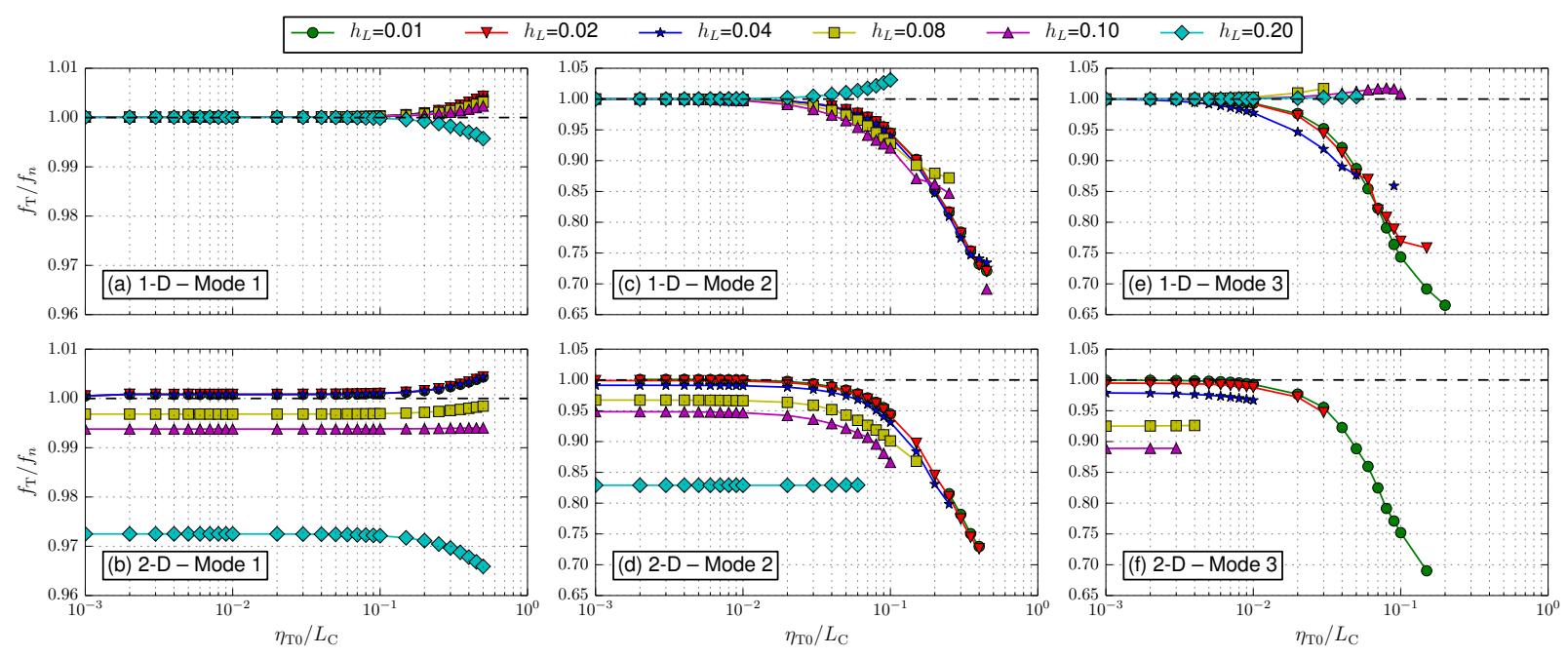

Figure 4: Analysis of the in vacuo dynamic response of the 1-D beam (top) and 2-D solid (bottom) models: Convergence of the cantilever oscillation frequency $f_{\mathrm{T}}$ to the linear theory as a function of the ratio between eigenmode deflection amplitude at the tip $\eta_{\mathrm{T} 0}$ and cantilever length $L_{\mathrm{C}}$ for several cantilever thickness-to-length ratios $h_{L}$, and Mode 1 (a,b), Mode 2 (c,d) and Mode 3 (e,f) shapes.

shape with a tip deflection $\eta_{\mathrm{T} 0}$. The results obtained for the static in vacuo model characterisation are summarised in Fig. 3. In the same way, the structural dynamic response is characterised by the frequency of the tip motion when the cantilever is released and oscillates free of any external load. The results obtained for the dynamic in vacuo model characterisation are summarised in Fig. 4.

For slender beam geometries, large bending deflections do not create large strains, so a linearised relation between stresses and strains is appropriate. In this case, both the two-dimensional solid model and the one-dimensional beam model, based on a geometrically non-linear theory, described in Section 2.2 can accurately capture the kinematics of the deformation for arbitrarily large displacements. For small displacements, the difference between these formulations and linear theory is negligible. The results presented in Figs. 3 and 4 confirm the expected modelling predictions. Thus, for the 1-D beam model and the relatively thin $\left(h_{L}<1 / 20\right) 2$-D solid model, the tip deflection $\eta_{\mathrm{T}}$ and oscilla- 

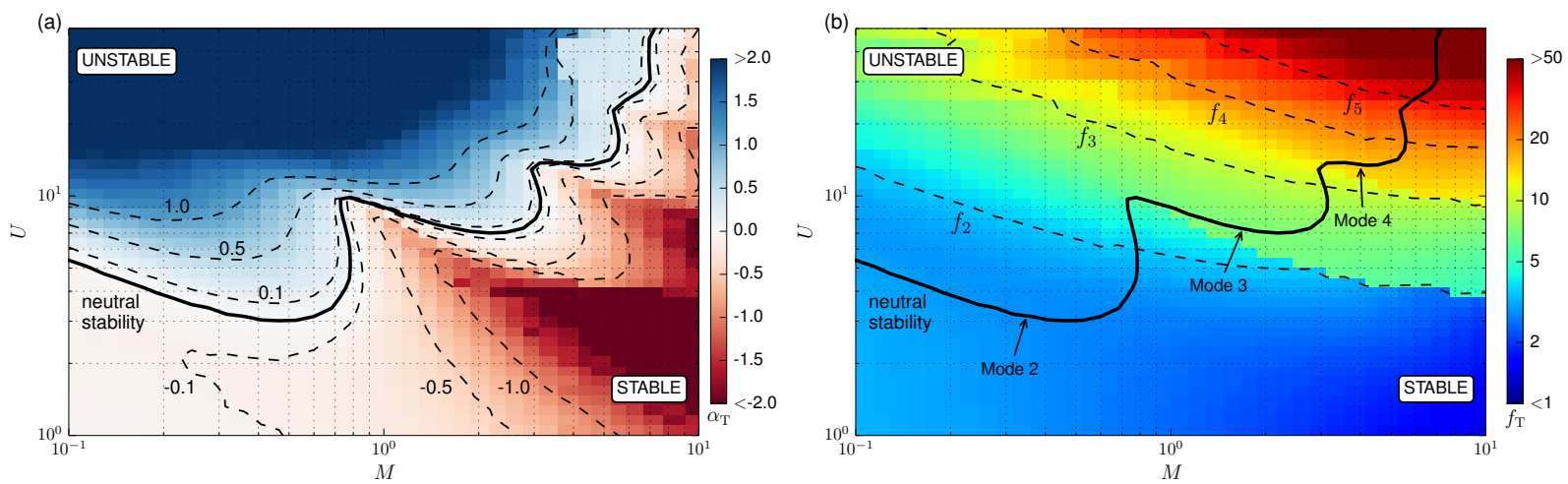

Figure 5: Characterisation of the stability of the FSI system containing the 1-D beam model for $L_{H}=2$ and $R e=100$ : (a) Exponential growth rate $\alpha_{\mathrm{T}}$ and (b) cantilever oscillation frequency $f_{\mathrm{T}}$ as functions of the mass ratio $M$ and reduced flow velocity $U$. The cantilever eigenfrequencies (in vacuo linear theory) associated with Modes 2 to 5 are indicated with dashed lines in (b).

tion frequency $f_{\mathrm{T}}$ predicted numerically converge well to the values given by linear theory for relatively small initial deflections $\left(\eta_{\mathrm{T} 0}<L_{\mathrm{C}} / 100\right)$. For increasing deflection amplitudes, a gradual increase in the differences between the linear and non-linear models predictions is observed. The size of these differences also becomes larger as the order of the structural mode gets higher.

As the thickness of the cantilever increases relative to its length, the predictions of $\eta_{\mathrm{T}}$ and $f_{\mathrm{T}}$ given by the 2-D solid model increasingly differ from those given by the geometrically non-linear and linear 1-D models. For relatively thick $\left(h_{L}>1 / 20\right)$ cantilevers, the slender beam approximation is not valid. Therefore, the differences between the predictions given by the 1-D and 2-D models become significant, particularly for the structural modes of order $n \geq 2$ and in the large displacement regime, i.e. as the initial tip deflection becomes larger. For particularly thick cantilevers, the predictions of the 2-D solid model differ considerably from those of the other models, and are not included in the results presented in Figs. 3 and 4 for the sake of clarity.

In what follows, the comprehensive analysis of the stability/instability of the FSI system is built on the 1-D geometrically non-linear beam model. The flexible cantilever is therefore chosen slender enough $\left(h_{\mathrm{C}}<L_{\mathrm{C}} / 50\right.$, see Table 1) for the effect of large displacements to be minimised. Also, the initial tip deflection is chosen small enough $\left(\eta_{\mathrm{T} 0}<L_{\mathrm{C}} / 200\right)$ for the simulation of the FSI system to start in the geometrically linear regime. However, this in vacuo characterisation shows that the simulation results are expected to be affected by the geometric non-linearity for growing large-amplitude cantilever motions, particularly for higher-order mode shapes.

\section{FSI system characterisation}

The methods described in Section 2.5 are now used to characterise the dynamics of the immersed flexible cantilever by its exponential growth/decay rate $\alpha_{\mathrm{T}}$ and frequency $f_{\mathrm{T}}$. This is undertaken for the combinations of mass ratio $M$ and reduced velocity $U$, listed in Table 1 , having fixed the flexible cantilever-length-to-channel-height ratio $L_{H}$ and Reynolds number $R e$; the variation of these latter two parameters is explored in subsequent sections. As indicated in Eq. (1), both non-dimensional parameters $U$ and $R e$ depend on the dimensional velocity $U^{*}$. Variations of the reduced velocity for a constant Reynolds number therefore correspond to variations of the cantilever flexural rigidity $B^{*}$ while keeping $U^{*}$ constant (see Section 8 for discussion). The values of $\alpha_{\mathrm{T}}$ and $f_{\mathrm{T}}$ estimated from the simulation data using the procedure detailed in Section 2.6 are consolidated to build the topography of the dynamic characteristics of the FSI system in the $(M, U)$ parameter space, as shown in Fig. 5 with fixed $L_{H}=2$ and $R e=100$. The solid line is the locus $\alpha_{\mathrm{T}}=0$ that represents the boundary between stable and unstable oscillations. The magnitude of $\alpha_{\mathrm{T}}$, represented by both marked contours and colour coding in Fig. 5(a), designates the strength of stability $\left(\alpha_{\mathrm{T}}<0\right)$ or instability $\left(\alpha_{\mathrm{T}}>0\right)$ of the FSI system. The neutral stability curve $\left(\alpha_{\mathrm{T}}=0\right)$ highlights the complex interactions of the flow with the different structural modes, the sequence of lobes indicating mode-switching from lower to higher order with increasing $M$. As mentioned in Section 2.6, the estimates of $\alpha_{\mathrm{T}}$ and $f_{\mathrm{T}}$ correspond to the mode dominating the 

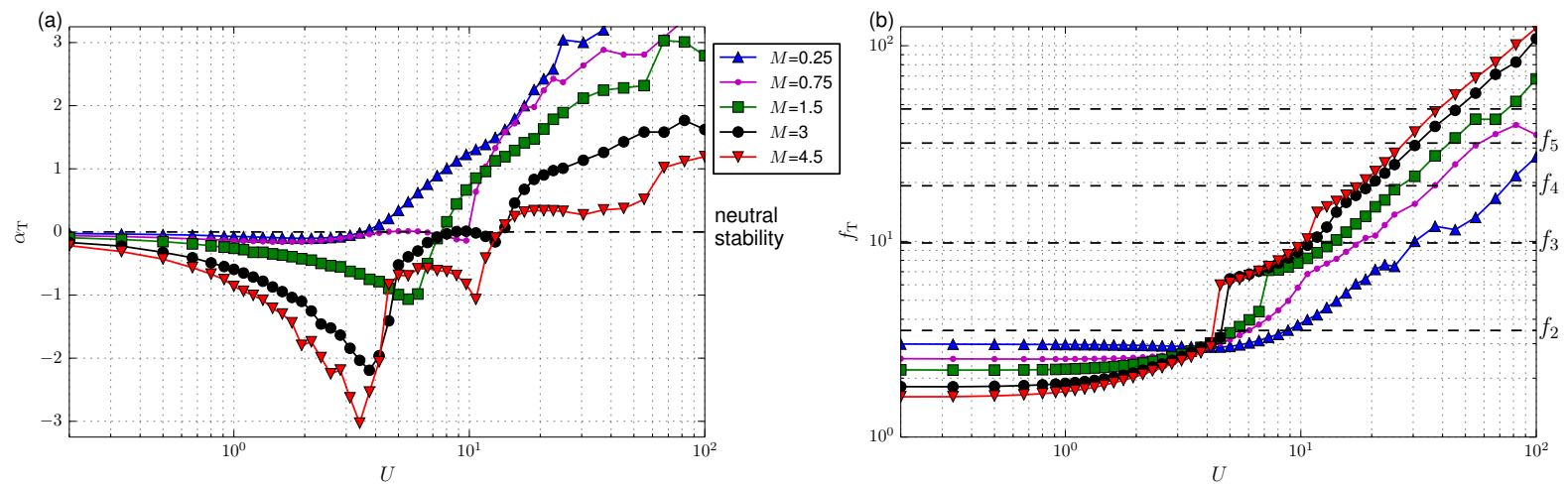

Figure 6: Characterisation of the stability of the FSI system containing the 1-D beam model for $L_{H}=2$ and $R e=100$ : (a) Exponential growth rate $\alpha_{\mathrm{T}}$ and (b) cantilever oscillation frequency $f_{\mathrm{T}}$ as a function of the reduced flow velocity $U$ for different mass ratio $M$. The cantilever eigenfrequencies (in vacuo linear theory) associated with Modes 2 to 5 are indicated with dashed lines in (b).

flexible-cantilever motion. Thus, the 'cascade' (lobe structure) topography in Fig. 5 can be understood as the result of the superposition of the topographies associated with the different modal motions, and represents the dynamic characteristics of the structural mode most excited by the flow for each parameter point $(M, U)$.

For low reduced velocities (typically $U<1$ ), the flow only has a very weak interaction with the flexible cantilever so that the latter oscillates as if in a viscous fluid at rest. Therefore, the initial Mode 2 deflection is damped in oscillatory motion. For $U \rightarrow 0$, the exponential decay rate of the oscillations converges to a very small value $\left(\alpha_{\mathrm{T}} \rightarrow\right.$ $0^{-}$). The frequency of the oscillations converges to a value lower than the in vacuo linear eigenfrequency associated with Mode 2 of the cantilever. This lower frequency can be linked to the $2^{\text {nd }}$ natural 'wet' frequency of an immersed cantilever, of which the motion is altered by the added mass of fluid $[10,16,42]$. This observation is consistent with results from earlier studies [10, 26, 31], which showed that fluid-inertia loading causes a decrease, primarily dependent upon the mass ratio, of the oscillation frequency of a structure in a fluid at rest, by comparison with the in vacuo case. The asymptotic convergence of $\alpha_{\mathrm{T}}$ and $f_{\mathrm{T}}$ for low reduced velocities can be seen more clearly in the curves shown in Fig. 6.

As the reduced velocity is increased from zero, the interaction of the flow with the flexible cantilever starts to take effect because the characteristic time scales of the structure and flow begin to match. The first effect, with increasing $U$, is modal stabilisation and mode-frequency increase for all mass ratios. These two trends can also be seen more clearly in the curves shown in Fig. 6. Stabilisation occurs because of irreversible energy transfer from the cantilever to the flow $[4,38]$. With further increases to $U$, the system behaviour undergoes a different change that is dependent upon the mass ratio. Figure 5 shows that, at low mass ratios $(M<0.8)$, Mode 2 remains the dominant structural mode and the frequency of the cantilever motion $f_{\mathrm{T}}$ monotonically increases. Having reached the minimum of low$U$ stabilisation effects, the value of $\alpha_{\mathrm{T}}$ then increases until it reaches 0 at the neutral stability boundary $U=U_{\text {crit }}$, marked by the solid line beyond which the energy transfer is from fluid flow to structure [4, 38]. For $U>U_{\text {crit }}$, both the frequency and exponential growth rate of the cantilever motion monotonically increase so that Mode 2 instability becomes stronger as $U$ increases. For higher mass ratios $(M>0.8)$, higher-order modes come into play. In a broader sense, increasing the mass ratio can be considered as reducing the mass per unit area of the structure for a given cantilever length and fluid density. This leads to higher natural frequencies of the structure requiring higher values of reduced velocity to match the characteristic time scales of structural dynamics and fluid flow. Alternatively, for fixed mass per unit area and fluid density, increasing $M$ can be interpreted as increasing the length of the cantilever, thereby promoting the presence of higher-order modes in the FSI. In this region, the initially imposed Mode 2 first dominates the cantilever motion and both frequency and exponential decay rate continue to increase with increasing $U$ until Mode 3 becomes the dominant structural mode at a sufficiently high value of $M$. Although the emergence of Mode 3 and the decline of Mode 2 actually overlap over a range of reduced velocities, this flutter-mode transition appears as a sharp discontinuity in $\alpha_{\mathrm{T}}$ and $f_{\mathrm{T}}$ in Fig. 5. For $0.8<M<3.2$, Mode 3 then becomes unstable after a pre-critical increase of $\alpha_{\mathrm{T}}$ and $f_{\mathrm{T}}$. For $M>3.2$, both frequency and exponential decay rate continue to increase with the increase 

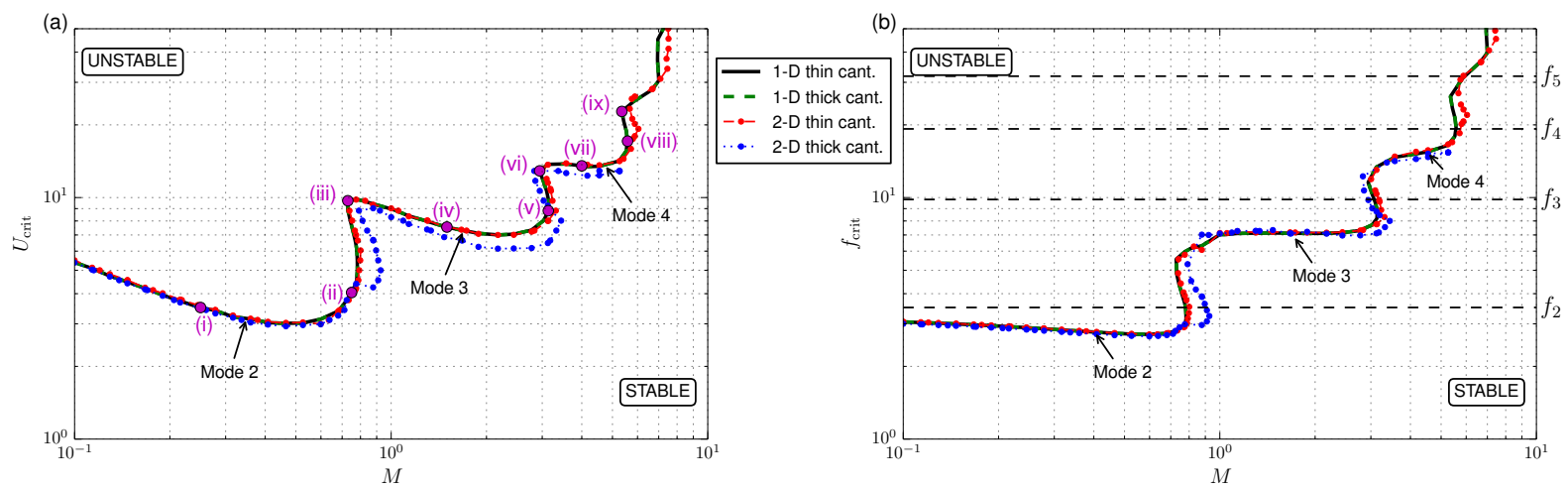

Figure 7: (a) Critical velocity $U_{\text {crit }}$ and (b) critical cantilever oscillation frequency $f_{\text {crit }}$ as functions of mass ratio $M$ for $L_{H}=2$ and $R e=100$, for the 1-D beam (see Fig. 5) and 2-D solid models. Thin and thick cantilevers denote $h_{L}=1 / 100$ and $h_{L}=1 / 20$, respectively. The cantilever eigenfrequencies (in vacuo linear theory) associated with Modes 2 to 5 are indicated with dashed lines in (b).

of reduced velocity, denoting the stabilisation of Mode 3, until Mode 4 becomes the dominant structural mode. Thus, for a given mass ratio, as the motion of the flexible cantilever becomes stabilised by the increase of reduced velocities when oscillating in lower-order mode shapes, then the next higher-order mode is successively excited by the flow, becoming the predominant flutter mode over a range of reduced velocity and thus creating the cascade structure seen in the neutral-stability curve. Thereafter, the order of the critical mode (that with the lowest $U_{\text {crit }}$ ) becomes higher as the mass ratio increases until the FSI system remains stable at any reduced velocity. In this region of very high $M$, the natural frequency of the structure is so high that increasingly high flow speeds are required for the destabilising fluid-structure interaction to occur; this can also be understood as the characteristic timescale of the structure being much smaller than that of the fluid flow. Overall, the predictions of the flexible-cantilever dynamic behaviour in the $(M, U)$ parameter space presented in Fig. 5 for $L_{H}=2$ and $R e=100$ agree qualitatively with the predictions of Shoele and Mittal [33] for a similar system with $L_{H}=1$ and $R e=400$. In the present study, the finer resolution of the (M, $U$ ) parameter space better elucidates the mode switching behaviour and provides more distinct stability boundaries.

The neutral stability curve obtained for $\alpha_{\mathrm{T}}=0$ as a function of reduced velocity and mass ratio has the classical shape seen in many previous studies [14, 15, 28, 34], including those that assume an inviscid fluid, with several modal branches. The critical velocity $U_{\text {crit }}$ and frequency $f_{\text {crit }}$ determined by the topography in Fig. 5 for $L_{H}=2$ and $R e=100$ are again shown in Fig. 7 as functions of the mass ratio. Also, the mode shapes of the flexible cantilever corresponding to particular points along the neutral stability curve are presented in Fig. 8. For points in the middle of the modal branches on the critical velocity curve, e.g. those designated (i,iv,vii) in Fig. 7(a), the flow excites a single structural mode, which becomes unstable for a well-defined $U_{\text {crit }}$. The shapes of the cantilever deflection seen in the first column of Fig. 8 are very close to the in vacuo eigenmode shapes. The corresponding $f_{\text {crit }}$ are comparable to the modal frequency with fluid added-mass, as shown in Fig. 7(b). However, for points located in the transition regions between modal branches, the flow interacts with two structural modes competing against each other. Thus in Fig. 8, the shapes corresponding to the points in the necks (e.g. (ii,v,viii)) and at the peaks (e.g. (iii,vi,ix)) of the critical velocity curve differ from the individual in vасио eigenmode shapes and illustrate the spatially-mixed modal motions of the flexible cantilever. For the particular mass ratios at which the modal transitions occur as the reduced velocity increases, e.g. $M=0.75$ with Modes 2 and 3, and $M=3$ with Modes 3 and 4, the competition between pure modal motions results first in a shallow instability followed by stability recovery and another instability, as shown in Fig. 6. This translates into several values of $U_{\text {crit }}$ for these particular mass ratios.

\section{FSI modelling and parameter sensitivity analysis}

The repercussions of the slender beam approximation within the structural modelling on the in vacuo static and dynamic responses of the flexible cantilever were discussed in Section 3. To complete the characterisation of the FSI system, the influence of the cantilever thickness-to-length ratio $h_{L}$ on the stability of the immersed cantilever is now 

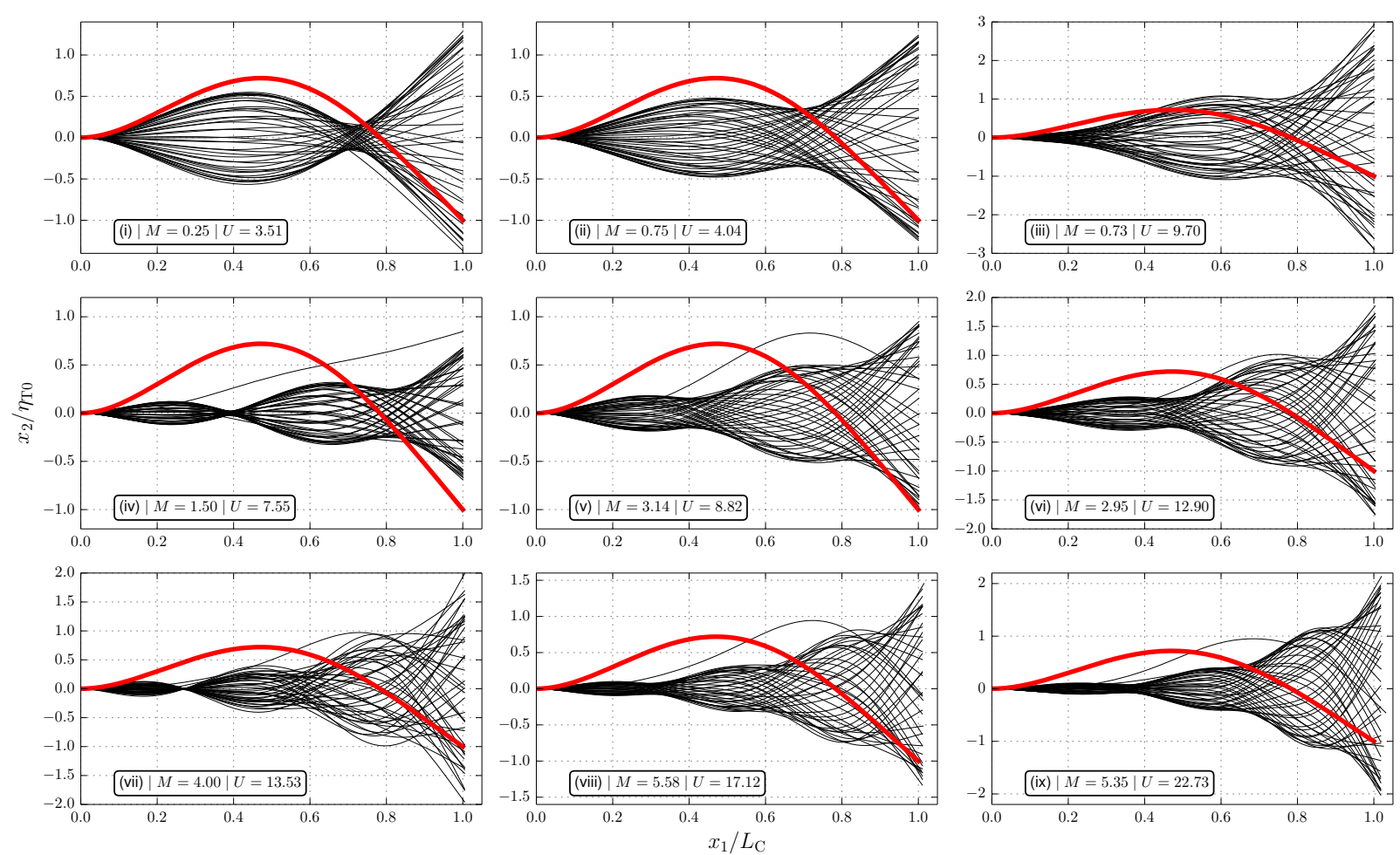

Figure 8: Mode shapes associated with the data points indicated in Fig. 7(a). Axial coordinates $x_{1}$ are scaled with the length of the flexible cantilever $L_{\mathrm{C}}$ and transverse coordinates $x_{2}$ are scaled with the initial transverse deflection of the flexible-cantilever tip $\eta_{\mathrm{T} 0}$. The thick red solid line shows the initial cantilever deflection.

analysed. Figure 7 shows the neutral stability curves corresponding to $L_{H}=2$ and $R e=100$ obtained with the 1-D beam and 2-D solid models for two $h_{L}$ ratios. As observed in the in vacuo characterisation, the thickness of the cantilever relative to its length has a negligible effect on the 1-D beam model dynamics. Therefore, the critical velocities and frequencies predicted with the 1-D beam model are only marginally affected by the change of thickness ratio. Thus, in Fig. 7, the critical velocity and frequency curves corresponding to the two values of $h_{L}$ are indistinguishable for the 1-D beam model. For the thin cantilever $\left(h_{L}=1 / 100\right)$, the neutral stability predictions obtained from the 1-D beam model only noticeably differ from those given by the 2-D solid model for $M>5$. Over this range of mass ratios, critical velocities and frequencies are high, and higher structural modes dominate the cantilever motion. For the thick cantilever $\left(h_{L}=1 / 20\right)$, however, the neutral stability predictions with the 1-D beam model are only close to those of the 2-D solid model for $M<0.8$, where Mode 2 dominates the cantilever motion. Moreover, for $M>5$, where modes of order $n>4$ start to become dominant as the reduced velocity increases, no critical properties can be estimated with the thick 2-D solid model. Due to limited localised-bending capabilities of the thick cantilever, the FSI system remains stable at any reduced velocity, for lower mass ratios in comparison to the thin 2-D solid model. The differences in stability prediction associated with the thick 2-D cantilever are explained in terms of changes in both the structural dynamics and the flow patterns. Firstly, as mentioned in Section 3, the slender beam approximation does not hold for relatively thick $\left(h_{L}>1 / 20\right)$ cantilevers. Greater differences are therefore observed between the steady deflections and in vacuo oscillation frequencies predicted by the 1-D beam and 2-D solid models, particularly for higher modes (see Figs. 3 and 4). Thus, the alteration of the structural dynamics has significant impact on the predictions of the immersed cantilever stability. Secondly, in the 1-D beam model, the thickness is only a parameter imparting the solid mechanical properties, not the geometric thickness. The 1-D beam included in the FSI system configuration is geometrically infinitely thin and the flow-conveying channel dimensions are constant for all the cantilever thicknesses considered. However, when the FSI system configuration includes the 2-D solid model, the thickness of the flexible cantilever modifies the system geometry by causing non-zero channel blockage. The thick cantilever is a bluff body 

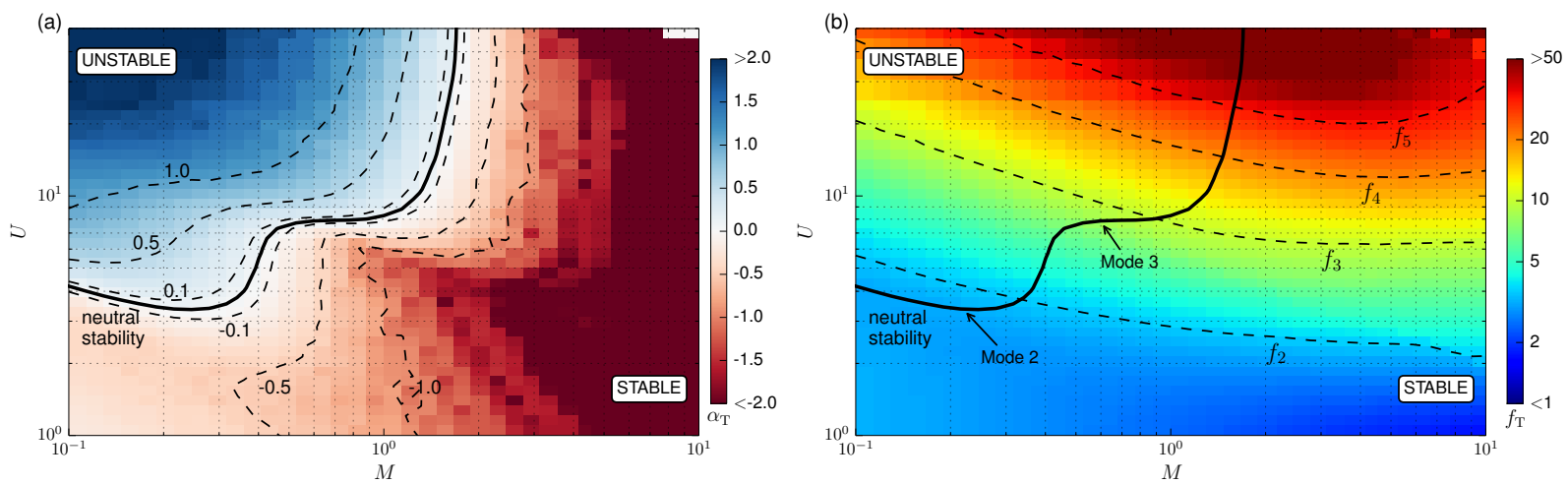

Figure 9: Characterisation of the stability of the FSI system containing the 1-D beam model for $L_{H}=2$ and $R e=10$ : (a) Exponential growth rate $\alpha_{\mathrm{T}}$ and (b) cantilever oscillation frequency $f_{\mathrm{T}}$ as functions of mass ratio $M$ and reduced flow velocity $U$. The cantilever eigenfrequencies (in vacuo linear theory) associated with Modes 2 to 5 are indicated with dashed lines in (b).

in the viscous flow field and a low-pressure region develops downstream of the blunt free end [12]. The obstruction of the channel and the altered flow properties play a role in the stability characteristics of the FSI system.

Finally, to ensure that the numerical parameters and the initial conditions controlling the simulations do not significantly influence the stability characterisation, a parametric sensitivity analysis of the FSI system implementation, based on the 1-D beam model, was carried out. This verification was made through the comparison of the exponential growth/decay rates $\alpha_{\mathrm{T}}$ and oscillation frequencies $f_{\mathrm{T}}$ obtained for the cases labelled (i) to (ix) on the stability curve shown in Fig. 7 with respect to variations of the following dimensional parameters: the height of the channel $H^{*}$, the length of the inlet rigid cantilever $L_{\text {inlet }}^{*}$, the distance from the cantilever free end to the outlet $L_{\text {outlet }}^{*}$, the thickness of the flexible cantilever $h_{\mathrm{C}}^{*}$, the initial tip deflection amplitude $\eta_{\mathrm{T} 0}^{*}$, the order of the mode used to initially deflect the flexible cantilever (see Section 2.3). Representative results of the parameter sensitivity analysis established that the variations of the control parameters and initial conditions have negligible effect on the FSI system stability characteristics. All the predicted near-critical frequency $f_{\mathrm{T}}$ data points for the cases denoted (i) to (ix) were almost superimposed. For the exponential growth/decay rates $\alpha_{\mathrm{T}}$, small discrepancies appeared depending on the case and the varied parameter/condition under consideration. However, all the data points fell within the range $-0.01<\alpha_{\mathrm{T}}<0.05$. Therefore, these discrepancies are marginal when compared to the range of $\alpha_{\mathrm{T}}$ values defining the stability topography of the FSI system presented in Fig. 5(a), in which the contours corresponding to $\alpha_{\mathrm{T}}=-0.1$ and $\alpha_{\mathrm{T}}=0.1$ are shown.

\section{Effect of Reynolds number}

In contrast to the many studies that assume inviscid flow, viscous effects are explicitly modelled in our stability analysis. The flow model based on the Navier-Stokes equation (2), including the terms corresponding to the viscous stresses, allows evaluation of the non-linear variations of added hydrodynamic mass, stiffness and damping [5] induced by the variations of Reynolds number and of their impact on the unsteady motion of the flexible cantilever. Figure 9 shows the topography of the FSI system dynamic characteristics in the $(M, U)$ parameter space for $L_{H}=2$ and $R e=10$. When compared to the results in Fig. 5 at $R e=100$, the drop in Reynolds number by one order of magnitude does not fundamentally modify the FSI mechanisms. The variations of exponential growth/decay rates $\alpha_{\mathrm{T}}$ and oscillation frequencies $f_{\mathrm{T}}$ appear to be globally similar. However, when compared to the $R e=100$ results, the features for $R e=10$ are (I) shifted to lower mass ratios $M$ because of the effect of the added mass, (II) stretched to higher reduced velocities $U$ because of the effect of the added stiffness, and (III) smoothed because of the effect of the added damping. Thus, the transition from Mode 2 to Mode 3 occurs for a lower mass ratio and is less abrupt. Also, all the structural modes of order greater than 3 are strongly stabilised and do not become the dominant response within the range of reduced velocities considered. The entire dynamics of the cantilever motion in the $(M, U)$ parameter space is therefore altered by the contribution of the viscous effects to the hydrodynamic forces exerted by the axial flow on the cantilever. 

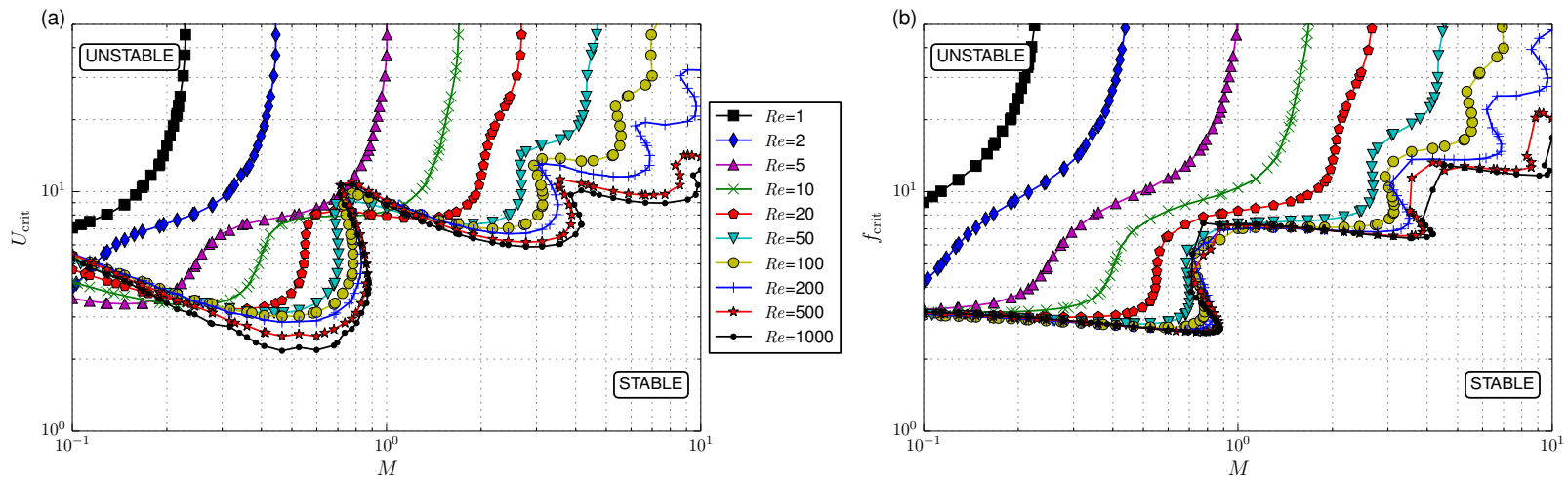

Figure 10: (a) Critical flow velocity $U_{\text {crit }}$ and (b) critical cantilever oscillation frequency $f_{\text {crit }}$ as functions of mass ratio $M$ for different Reynolds numbers $R e$ and $L_{H}=2$.
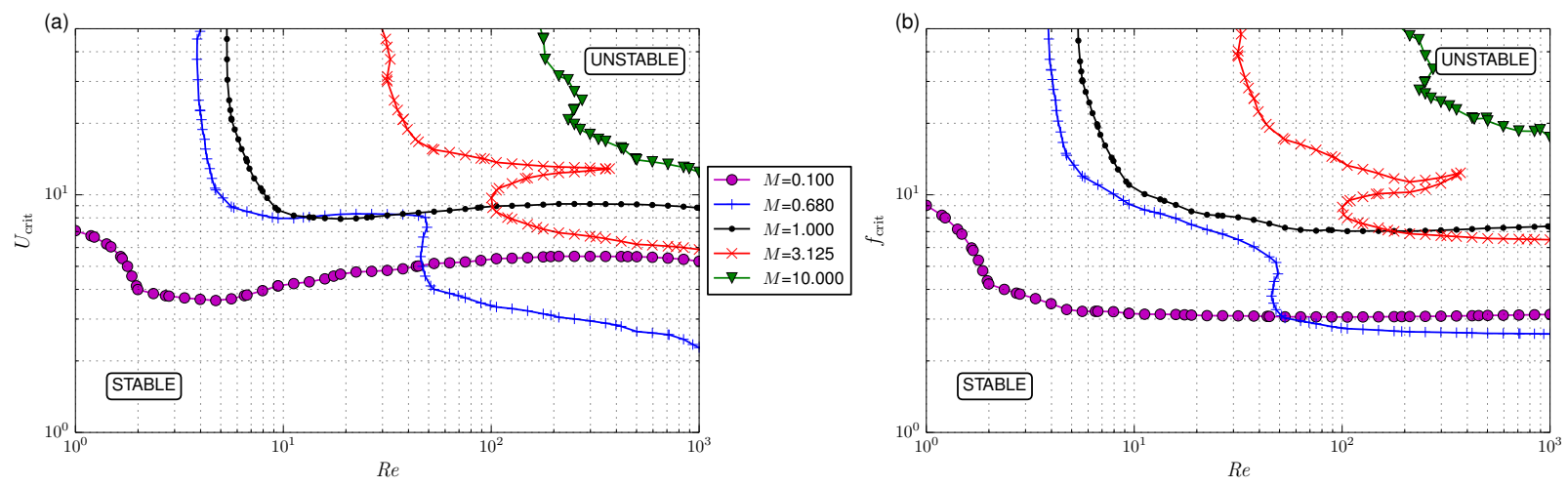

Figure 11: (a) Critical flow velocity $U_{\text {crit }}$ and (b) critical cantilever oscillation frequency $f_{\text {crit }}$ as functions of Reynolds number $R e$ for different mass ratios $M$ and $L_{H}=2$.

Figure 10(a) shows the lines of reduced critical velocity for a range of Reynolds numbers. It can be seen that the modes of order higher than 3 are successively stabilised when the Reynolds number decreases from 1000 to 20 . Similarly, the cascade structure of the instability is gradually lost so that, at the lowest Reynolds numbers, only one structural mode can potentially become unstable for a given mass ratio. For $R e<20$, only Mode 2 and Mode 3 become unstable for the entire range of mass ratios considered. For these two modes, the critical velocities remain of the same order of magnitude but are shifted to lower mass ratios with the decrease in Reynolds number. Therefore, the range of mass ratios for which the FSI system remains stable at any reduced velocity increases as the Reynolds number decreases.

As shown in Fig. 10(b), the decrease in Reynolds number also produces an increase in the critical frequencies for a given mass ratio. This change in frequency is due to the higher mode instabilities being triggered at lower mass ratios as the Reynolds number decreases. It also results from the immersed cantilever oscillating at frequencies closer to the cantilever natural frequencies for near-critical conditions at lower Reynolds number, as a comparison of Fig. 5(b) and Fig. 9(b) shows. However, Figure 10(b) indicates that the frequency of the immersed cantilever motion converges to the same $2^{\text {nd }}$ 'wet' frequency of the cantilever as the mass ratio tends to zero irrespective of the Reynolds number.

The present comprehensive analysis of the Reynolds number dependence of the FSI system dynamics and hence of its stability boundaries extends previous investigations of a flexible cantilever immersed in a viscous channel flow $[4,33,38]$. The FSI system dynamic characteristics in the $(M, U)$ parameter space exhibit non-linear transformations associated with the variation of Reynolds number. As a consequence, the critical reduced velocity curves corresponding to different Reynolds numbers can intersect, as shown in Fig. 10(a). This leads to ranges of mass ratios for which the evolution of the critical reduced velocity with respect to the Reynolds number becomes non-monotonic, as shown 

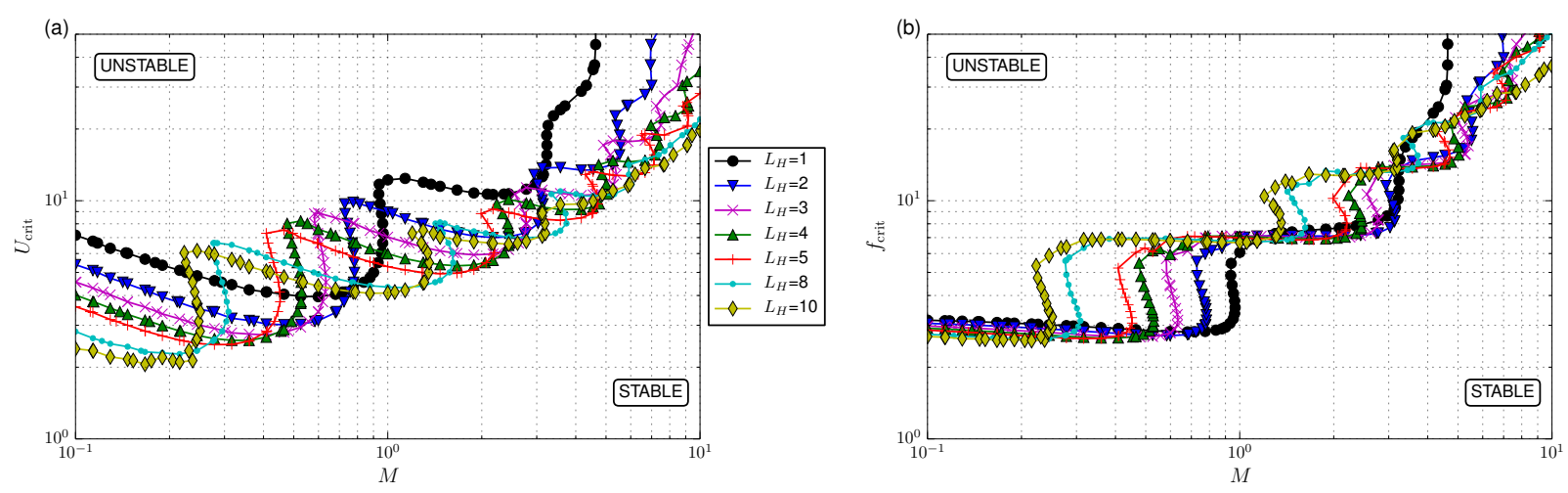

Figure 12: (a) Critical flow velocity $U_{\text {crit }}$ and (b) critical cantilever oscillation frequency $f_{\text {crit }}$ as functions of the mass ratio $M$ for different cantilever-length-to-channel-height ratios $L_{H}$ and $R e=100$.

in Fig. 11(a). For particular mass ratios, e.g. $M=0.1$ and $M=1.0$, the critical flow velocity can decrease as the Reynolds number decreases before reaching a minimum and then increasing. This suggests that an additional instability mechanism related to the wall shear stress and the cantilever axial stretch might be occurring at low Reynolds numbers, as that occurs in viscous flow over compliant walls [32, 39]. For other particular mass ratios, e.g. $M=0.68$ and $M=3.125$, the Reynolds number decrease induces a change in the first unstable structural mode and hence a jump in critical oscillation frequency, as shown in Fig. 11(b). For most mass ratios however, the critical velocity simply increases as the Reynolds number decreases and the first unstable structural mode remains identical.

The present analysis done on a two-dimensional system discounts any three-dimensional effects, which are known to influence the stability of immersed rectangular plates. It has been shown that, in general, a reduction of the span-tolength ratio of plates in inviscid flow stabilises the FSI system $[14,17]$, particularly for low mass ratios. Further, using full three-dimensional FSI simulations based on the immersed boundary method, Huang and Sung [24] have shown that, for a flag with a span-to-length ratio of unity, viscous forces could induce spanwise bending near the free trailing edge. For increased Reynolds numbers, this spanwise bending could see its amplitude reduced but could become asymmetric. For plates with a span larger than the length, the effects of the finiteness of the span on the flexible solid motion and on the stability boundary of the FSI system remain limited, so that the present two-dimensional results can be considered representative of these system configurations.

\section{Effect of cantilever confinement}

The stability of the flexible cantilever is greatly affected by the channel walls [6]. Several studies of the equivalent inviscid flow model [18, 22], and physical measurements [2, 34], have shown that for channels narrowed down to a height smaller than the cantilever length, the FSI system becomes more unstable than for a flexible cantilever immersed in an unbounded flow domain. The drop in critical velocity when $L_{H}>1$ has been attributed to supplementary inertial effects resulting from the channel narrowing associated with the Bernoulli effect and mass conservation, and generating a higher pressure difference across the flexible cantilever.

Unlike FSI models based on inviscid flow, the model used in this study allows characterisation of the effects of the pressure losses produced by viscous forces on the flexible cantilever stability. The contribution of this viscosity-related pressure component depends primarily on the Reynolds number. However, it is also affected by the confinement of the cantilever, accounted for by the cantilever-length-to-channel-height ratio $L_{H}$. Figure 12 therefore compares the critical velocity and frequency curves obtained at a fixed Reynolds number $R e=100$ for different values of $L_{H}$. Increases in relative length of the cantilever $L_{H}$ produce a global lowering of the critical velocities, in a way similar to that observed with potential-flow based modelling $[18,34]$. This destabilisation of the FSI system as the flexible cantilever becomes relatively more confined in the channel is accompanied by a shift of the modal transitions to lower mass ratios. However, the shape of the branches of the critical velocity curves corresponding to Mode 2 and Mode 3 remains similar as the value of $L_{H}$ varies. This indicates that the effect of the viscosity-related pressure on 

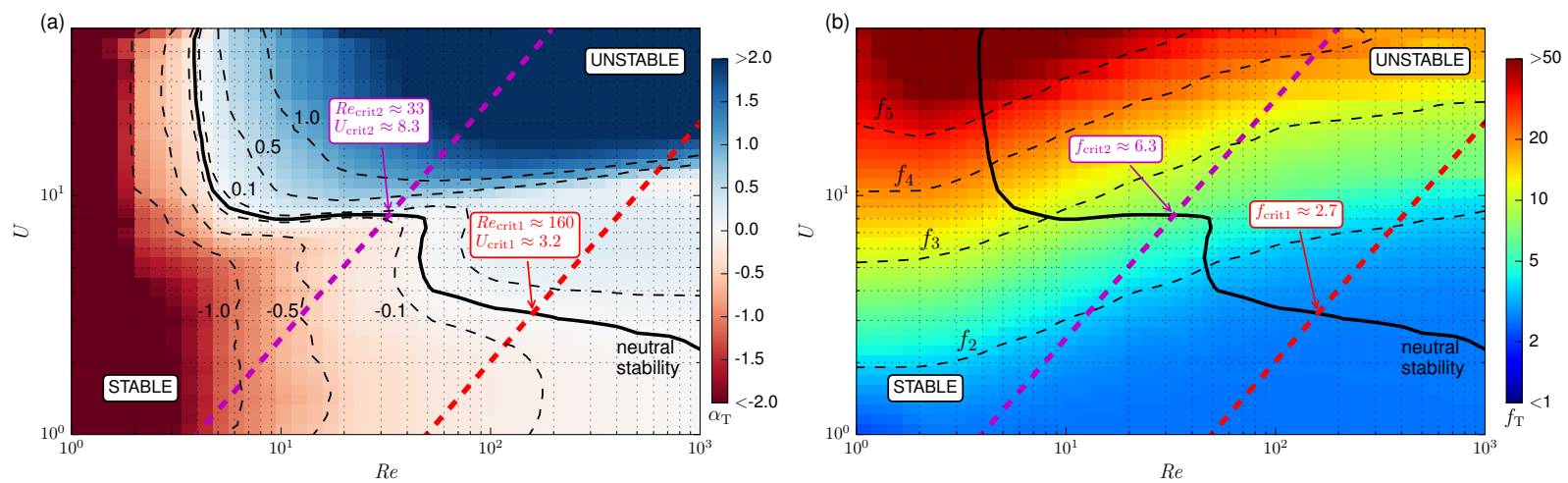

Figure 13: Characterisation of the stability of the FSI system containing the 1-D beam model for $L_{H}=2$ and $M=0.68$ : (a) Exponential growth rate $\alpha_{\mathrm{T}}$ and (b) cantilever oscillation frequency $f_{\mathrm{T}}$ as functions of Reynolds number $R e$ and reduced flow velocity $U$. The cantilever eigenfrequencies (in vacuo linear theory) associated with Modes 2 to 5 are indicated with thin dashed lines in (b). The thick dash lines and indicated critical values correspond to the two cases discussed in Section 8.

the system dynamics remains largely independent of the confinement effect for the lower-mode instabilities, which are characterised by low-curvature mode shapes. On the other hand, the stabilisation of the higher modes $(n>3)$ associated with decreasing Reynolds numbers, discussed in Section 6, is diminished when the flexible cantilever becomes much longer than the channel height. The relative narrowing of the channel increases the importance of the flow inertial effects over the viscous effects even if the Reynolds number remains constant. These stronger inertial effects also result in a sharpening of the modal transitions along the neutral stability curves. Finally, it can be seen from Fig. 12(b) that the critical frequency curves are only affected by the shift of the modal transitions to lower mass ratios. This reveals that cantilever-confinement does not significantly alter the immersed cantilever motion frequency, which converges to the same $2^{\text {nd }}$ 'wet' frequency of the cantilever as the mass ratio tends to zero irrespective of the cantilever-length-to-channel-height ratio.

\section{Illustrative physical systems}

In the vast majority of physical systems, the geometric configuration and the cantilever mechanical properties are known while the velocity of the fluid flowing in the channel changes. Therefore, the non-dimensional $U$ and $R e$ would both vary linearly with the dimensional $U^{*}$ while the non-dimensional $L_{H}$ and $M$ would remain constant. Figure 13 shows the topography of the FSI system dynamic characteristics in the $(R e, U)$ parameter space for $L_{H}=2$ and $M=0.68$. To illustrate the effect of the fluid viscosity on the properties of the neutral stability of the FSI system in configurations corresponding to this particular $\left(L_{H}, M\right)$ pairing, two cases involving flexible cantilevers made of the same material $\left(\rho_{\mathrm{C} 1}^{*}=\rho_{\mathrm{C} 2}^{*}, E_{1}^{*}=E_{2}^{*}\right.$ and $\left.v_{1}=v_{2}\right)$ and inserted in a channel of same height $\left(H_{1}^{*}=H_{2}^{*}\right)$ are considered. In the first case, the fluid flowing in the channel has a density $\rho_{\mathrm{F} 1}^{*}$ and a dynamic viscosity $\mu_{\mathrm{F} 1}^{*}$, and in the second case, the fluid has a density $\rho_{\mathrm{F} 2}^{*}=\rho_{\mathrm{F} 1}^{*} / 5$ and a dynamic viscosity $\mu_{\mathrm{F} 2}^{*}=\mu_{\mathrm{F} 1}^{*} / 2$. The mass ratio is kept constant $\left(M_{1}=M_{2}\right)$ by changing the thickness of the flexible cantilever so that $h_{\mathrm{L} 2}=h_{\mathrm{L} 1} / 5$. Both $h_{\mathrm{L} 1}$ and $h_{\mathrm{L} 2}$ remain within the range of thickness where the slender beam approximation holds. For both cases, an inviscid FSI model would predict an identical critical reduced velocity $U_{\text {crit }}$ and an identical critical oscillation frequency $f_{\text {crit }}$ with a Mode 2 cantilever motion. Indeed, the prediction of these two critical values would only be determined by the value of the mass ratio, identical in both cases. By contrast, the present FSI model that explicitly accounts for viscous effects predicts substantially different values of the critical state for the two cases. As shown in Fig. 13, the 'trajectories' in the $(R e, U)$ parameter space, with respect to the variation of the inlet flow velocity $U^{*}$, corresponding to the two cases, cross the neutral stability curve in two different locations. Thus, the FSI system is predicted to become unstable for a reduced velocity $U_{\text {crit } 1} \approx 3.2$ for the first fluid, and for $U_{\text {crit } 2} \approx 8.3$ for the second fluid. Therefore, the FSI system is considerably more stable in the second case. Moreover, as shown in Fig. 13(b), it is predicted that the neutral stability occurs for a Mode 2 cantilever oscillation for the first fluid but for a Mode 3 cantilever oscillation for the second fluid. 
This translates into a critical oscillation frequency for the second case $\left(f_{\text {crit } 2} \approx 6.3\right)$ being more than twice that for the first case $\left(f_{\text {crit1 }} \approx 2.7\right)$.

\section{Conclusions}

A numerical model coupling the Navier-Stokes equations and a one-dimensional elastic Kirchhoff-Love beam model has been employed to characterise the dynamics of a flexible cantilever immersed in viscous channel flow. Systematic verifications of the implementation of this FSI model and comparisons of its predictions with those of a FSI model containing a two-dimensional solid model have been carried out to ensure reliable simulations of the flow-induced motion of a slender cantilever. The comprehensive parametric analysis presented revealed the different effects of viscosity on the single-mode flutter instability of the FSI system and consequently on the critical velocity and frequency values.

In general, the FSI system is shown to become more stable as the Reynolds number decreases, in particular for high fluid-to-solid mass ratios. A drop in Reynolds number from $R e=1000$ by several orders of magnitude does not fundamentally modify the FSI mechanism. However, the predicted changes to the dynamics of the immersed flexible cantilever, arising from the increased contribution of the viscous effects to the hydrodynamic forces, can lead to large variations of the neutral stability characteristics.

The shape of the critical velocity curves, characterised by a cascade of modal branches with increasing mass ratio, undergoes significant changes in its features as the viscous effects within the flow become more dominant. Thus, a sufficient decrease of the Reynolds number can lower the critical velocity and produce a change in the first unstable structural mode. The confinement of the cantilever tends to increase the importance of the flow inertial effects relative to the viscous effects even if the Reynolds number remains constant.

These findings warrant future detailed investigations of the instability mechanisms at very low Reynolds number. Finally, the comprehensive stability data presented in this paper can be used to estimate dimensional critical flutter speeds and oscillation frequencies for a wide range of channel-immersed-cantilever systems found in engineering applications.

\section{Acknowledgement}

The authors gratefully acknowledge the support of the Australian Research Council through project DP0559408 and the WA State Centre of Excellence in eMedicine through project 'Airway Tomography Instrumentation'. This work was supported by resources provided by The Pawsey Supercomputing Centre with funding from the Australian Government and the Government of Western Australia.

\section{References}

[1] L. Auman and B. Wilks. Application of fabric ribbons for drag and stabilization. In 18th AIAA Aerodynamic Decelerator Systems Technology Conference and Seminar, 2005.

[2] Y. Aurégan and C. Depollier. Snoring: linear stability analysis and in-vitro experiments. J Sound Vib, 188:39-53, 1995.

[3] J. Backus. Small-vibration theory of the clarinet. J Acoust Soc Am, 35:305-313, 1963.

[4] T. S. Balint and A. D. Lucey. Instability of a cantilevered flexible plate in viscous channel flow. J Fluid Struct, 20:893-912, 2005.

[5] R. A. Bidkar, M. Kimber, A. Raman, A. Bajaj, and S. V. Garimella. Nonlinear aerodynamic damping of sharp-edged flexible beams oscillating at low keulegan-carpenter numbers. J Fluid Mech, 634:269-289, 2009. doi: 10.1017/S0022112009007228.

[6] C. E. Brennen. A review of added mass and fluid inertial forces. Technical report, Naval Civil Engineering Laboratory, Department of the Navy, 1982.

[7] J. Cisonni, N. S. J. Elliott, A. D. Lucey, and M. Heil. A compound cantilevered plate model of the palate-uvula system during snoring. In H. Chowdhury and F. Alam, editors, 19th Australasian Fluid Mechanics Conference, Melbourne, Australia, Dec 8 2014. RMIT University.

[8] J. Cisonni, A. D. Lucey, and N. S. J. Elliott. Stability of a cantilevered flexible plate with non-uniform thickness in viscous channel flow. In Y. Zhou, A.D. Lucey, Y. Liu, and L. Huang, editors, Proceedings of the 3rd Symposium on Fluid-Structure-Sound Interactions and Control. Springer, 2016. doi: 10.1007/978-3-662-48868-3.

[9] B. S. H. Connell and D. K. P. Yue. Flapping dynamics of a flag in a uniform stream. J Fluid Mech, 581:33-67, 2007. doi: 10.1017/ S0022112007005307.

[10] E. Da Lozzo, F. Auricchio, and G. M. Calvi. Added mass model for vertical circular cylinder partially immersed in water. In Proceedings of 15th WCEE, Lisboa, 2012. 
[11] O Doaré, M Suazade, and C Eloy. Flutter of an elastic plate in a channel flow: Confinement and finite-size effects. J Fluid Struct, 27:76-88, 2011. doi: 10.1016/j.jfluidstructs.2010.09.002.

[12] N. S. J. Elliott, A. D. Lucey, and M. Heil. Large-amplitude oscillations of a finite-thickness cantilevered flexible plate in viscous channel flow. In 3rd Joint US-European Fluids Engineering Summer Meeting and 8th International Conference on Nanochannels, Microchannels and Minichannels (FEDSM-ICNMM2010), pages 287-295, Montreal, Canada, Aug 1 2010. American Society of Mechanical Engineers (ASME).

[13] N. S. J. Elliott, A. D. Lucey, M. Heil, P. R. Eastwood, and D. R. Hillman. Modelling and simulation of fluid-structure interactions in human snoring. In F. Chan, D. Marinova, and R. S. Anderssen, editors, 19th International Congress on Modelling and Simulation (MODSIM 2011), pages 530-536, Perth, Australia, Dec 12 2011. Modelling and Simulation Society of Australia and New Zealand Inc.

[14] C. Eloy, C. Souilliez, and L. Schouveiler. Flutter of a rectangular plate. J Fluid Struct, 23:904-919, 2007. doi: 10.1016/j.jfluidstructs.2007. 02.002 .

[15] C. Eloy, R. Lagrange, C. Souilliez, and L. Schouveiler. Aeroelastic instability of cantilevered flexible plates in uniform flow. J Fluid Mech, 611:97-106, 2008. doi: 10.1017/S002211200800284X.

[16] Y. Fu and W. G. Price. Interactions between a partially or totally immersed vibrating cantilever plate and the surrounding fluid. J Sound Vib, 118:495-513, 1987.

[17] S. C. Gibbs, I. Wang, and E. Dowell. Theory and experiment for flutter of a rectangular plate with a fixed leading edge in three-dimensional axial flow. J Fluid Struct, 34:68-83, 2012. doi: 10.1016/j.jfluidstructs.2012.06.009.

[18] C. Q. Guo and M. P. Païdoussis. Stability of rectangular plates with free side-edges in two-dimensional inviscid channel flow. J Appl Mech, 67:171-176, 2000

[19] M. Heil and A. L. Hazel. oomph-lib - an object-oriented multi-physics finite-element library. In M. Schäfer and H.-J. Bungartz, editors, Fluid-Structure Interaction, pages 19-49. Springer, 2006. oomph-1ib and its extensive documentation are freely available as open-source software at http://www.oomph-lib.org.

[20] M. Heil, A. L. Hazel and J. Boyle. Solvers for large-displacement fluid-structure interaction problems: Segregated vs. monolithic approaches. Comput Mech, 43:91-101, 2008.

[21] R. M. Howell and A. D. Lucey. Flutter of spring-mounted flexible plates in uniform flow. J Fluid Struct, 59:370-393, 2015. doi: 10.1016/j. jfluidstructs.2015.09.009.

[22] R. M. Howell, A. D. Lucey, P. W. Carpenter, and M. W. Pitman. Interaction between a cantilevered-free flexible plate and ideal flow. J Fluid Struct, 25:544-566, 2009.

[23] L. Huang. Flutter of cantilevered plate in axial flow. J Fluid Struct, 9:127-147, 1995.

[24] W.-X. Huang and H. J. Sung. Three-dimensional simulation of a flapping flag in a uniform flow. J Fluid Mech, 653:301-336, 2010. doi: $10.1017 / \mathrm{S} 0022112010000248$.

[25] S. F. Kistler and L. E. Scriven Coating flows. In J. R. A. Pearson and S. M. Richardson, editors, Computational analysis of polymer processing, pages 243-299. Applied Science Publishers, London, 1983.

[26] A. Kornecki, E. H. Dowell, and J. O'Brien. On the aeroelastic instability of two-dimensional panels in uniform incompressible flow. J Sound Vib, 47:163-178, 1976.

[27] S. Mandre and L. Mahadevan. A generalized theory of viscous and inviscid flutter. Proc R Soc A, 466:141-156, 2010. doi: 10.1098/rspa. 2009.0328.

[28] S. Michelin, S. G. Llewellyn Smith, and B. J. Glover. Vortex shedding model of a flapping flag. J Fluid Mech, 617:1-10, 2008. doi: 10.1017/S0022112008004321.

[29] M. P. Païdoussis. Fluid-Structure Interactions: Slender Structures and Axial Flow, volume 1. Academic Press, London, 1998.

[30] M. P. Païdoussis. Fluid-Structure Interactions: Slender Structures and Axial Flow, volume 2. Academic Press, London, 2003.

[31] R. Shabani, H. Hatami, F. G. Golzar, S. Tariverdilo, and G. Rezazadeh. Coupled vibration of a cantilever micro-beam submerged in a bounded incompressible fluid domain. Acta Mech, 224:881-850, 2013. doi: 10.1007/s00707-012-0792-z.

[32] V. Shankar and V. Kumaran. Stability of wall modes in fluid flow past a flexible surface. Phys Fluids, 14(7):2324-2338, 2002.

[33] K. Shoele and R. Mittal. Computational study of flow-induced vibration of a reed in a channel and effect on convective heat transfer. Phys Fluids, 26, 2014. doi: 10.1063/1.4903793.

[34] K. Shoele and R. Mittal. Flutter instability of a thin flexible plate in a channel. J Fluid Mech, 786:29-46, 2016. doi: 10.1017/jfm.2015.632.

[35] L. Tang and M. P. Païdoussis. On the instability and the post-critical behaviour of two-dimensional cantilevered flexible plates in axial flow. J Sound Vib, 305:97-115, 2007. doi: 10.1016/j.jsv.2007.03.042.

[36] L. Tang and M. P. Païdoussis. The influence of the wake on the stability of cantilevered flexible plates in axial flow. J Sound Vib, 310: 512-526, 2008. doi: 10.1016/j.jsv.2007.09.025.

[37] L. Tang, M. P. Païdoussis, and J. Jiang. Cantilevered flexible plates in axial flow: Energy transfer and the concept of flutter-mill. J Sound Vib, 326:263-276, 2009. doi: 10.1016/j.jsv.2009.04.041

[38] G. A. Tetlow and A. D. Lucey. Motions of a cantilevered flexible plate in viscous channel flow driven by a constant pressure drop. Commun Numer Meth En, 25:436-482, 2009.

[39] K. Tsigklifis and A. D. Lucey. Global stability analysis of blasius boundary-layer flow over a compliant panel accounting for axial and vertical displacements. In Y. Zhou, A. D. Lucey, Y. Liu, and L. Huang, editors, Fluid-Structure-Sound Interactions and Control Proceedings of the 3rd Symposium on Fluid-Structure-Sound Interactions and Control, Lecture Notes in Mechanical Engineering, pages 357-362. Springer, 2016.

[40] E. Virot, X. Amandolese, and P. Hémon. Fluttering flags: An experimental study of fluid forces. J Fluid Struct, 43:385-401, 2013. doi: 10.1016/j.jfluidstructs.2013.09.012.

[41] Y Watanabe, K Isogai, S Suzuki, and M Sugihara. A theoretical study of paper flutter. J Fluid Struct, 16:543-560, 2002. doi: 10.1006/jfls. 2001.0436.

[42] Y. Yadykin, V. Tenetov, and D. Levin. The added mass of a flexible plate oscillating in a fluid. J Fluid Struct, 17:115-123, 2003. 